\title{
ORGANOTIN(IV) COMPLEXES OF SCHIFF BASES: A REVIEW
}

\author{
Mala Nath* and Savita Goyal \\ Chemistry Department, University of Roorkee, Roorkee - 247667 India
}

\section{TABLE OF CONTENTS}

\subsection{INTRODUCTION}

\subsection{SYNTHESIS}

\subsubsection{Adducts}

1.2.2 Substitution Derivatives

1.2.2.1 Deprotonation with triethylamine

1.2.2.2 Template synthesis

1.2.2.3 By the sodium salt method

1.2.2.4 By direct $\mathrm{Sn}-\mathrm{Cl}$ bond cleavage reaction using silylated compounds

1.2.2.5 By the potassium salt method

1.2.2.6 By the thallium salt method

1.2.2.7 By ligand exchange

1.2.2.8 By the reaction of organotin(IV) amides with Schiff bases

1.2.2.9 By the reaction of trialkyltin(IV) alkoxides with Schiff bases

1.2.2.10 By the reactions of trialkyltin hydroxides and Schiff bases

1.2.2.11 By the reaction of diorganotin(IV) oxides with Schiff bases

1.3 PROPERTIES

1.3.1 Hydrolytic stability

1.3.2 Volatility and Thermal Stability

13.3 Molecular Association

1.4 STRUCTURAL STUDIES

1.4.1 Molar Conductance

1.4.2 Electronic Spectra

1.4.3 Infrared Spectra

1.4.4 Nuclear $\left({ }^{1} \mathrm{H},{ }^{13} \mathrm{C}\right.$ and $\left.{ }^{119} \mathrm{Sn}\right)$ magnetic resonance spectral studies

1.4.5 ${ }^{119} \mathrm{Sn}$ Mössbauer and X-ray crystallographic studies

1.5 Mass Spectral Studies

1.6 Thermal Studies

1.7 Antimicrobial Activity 


\section{LIST OF ABBREVIATIONS}

\section{CHEMICALS, LIGANDS AND SOLVENTS}

$\mathrm{CD}_{3} \mathrm{OD}$

$\mathrm{CDCl}_{3}$

$\mathrm{CD}_{2} \mathrm{Cl}_{2}$

DMSO

DMSO- $_{6}$

DMF

DMA

$\mathrm{H}_{2}$ acen

$\mathrm{H}_{2} \mathrm{acpn}$

$\mathrm{H}_{2}$ aceten

$\mathrm{H}_{2}$ acetdap

$\mathrm{H}_{2}$ salen

$\mathrm{H}_{2}$ salpn

$\mathrm{H}_{2}$ salaen

$\mathrm{H}_{2}$ saldap-2-ol

$\mathrm{H}_{2}$ saladap-2-ol

$\mathrm{H}_{2}$ salphen-o

$\mathrm{H}_{2}$ salap

$\mathrm{H}_{2}$ salatp

$\mathrm{Htdbm} \mathrm{Pr}$

$\mathrm{H}_{2}$ vanilen

$\mathrm{H}_{2}$ salcen

HMPA

THF

TDPU
Deuterated methanol

Deuterated chloroform

Deuterated dichloromethane

Dimethyl sulfoxide

Deuterated dimethyl sulfoxide

$\mathrm{N}, \mathrm{N}$-dimethylformamide

Dimethylacetamide

$\mathrm{N}, \mathrm{N}$-Ethylenebis(acetylacetoneimine)

$\mathrm{N}, \mathrm{N}$-Propylenebis(acetylacetoneimine)

$\mathrm{N}, \mathrm{N}$ '-Ethylenebis(2-hydroxyacetophenoneimine)

$\mathrm{N}, \mathrm{N}$ '-Trimethylenebis(2-hydroxyacetophenoneimine)

$\mathrm{N}, \mathrm{N}$-Ethylenebis(salicylaldimine)

$\mathrm{N}, \mathrm{N}$-Propylenebis(salicylaldimine)

Bis(salicylic acid)ethylenediimine

$\mathrm{N}, \mathrm{N}^{\prime}$-2-Hydroxytrimethylenebis(salicylaldimine)

Bis(salicylic acid)-2-hydroxytrimethylenediimine)

$\mathrm{N}, \mathrm{N}^{\prime}-o$-Phenylenebis(salicylaldimine)

$\mathrm{N}-2$-Hydroxyphenylsalicylaldimine

2-(2-Hydroxyphenyl)benzothiazoline

Thiodibenzoylmethane-i-propylimine

Bis(vanillin)ethylenediimine

$\mathrm{N}, \mathrm{N}$-Ethylenebis(3-carboxysalicylaldimine)

Hexamethylphosphoramide

Tetrahydrofuran

Trimethylsilyl-N-N'-diphenylurea 


\begin{abstract}
A comprehensive review on organotin(IV) complexes of Schiff bases is presented with special reference to their methods of synthesis, structural and thermal properties as well as their biological activity. The structures of these complexes are discussed on the basis of IR, electronic, multinuclear magnetic $\left({ }^{1} \mathrm{H},{ }^{13} \mathrm{C}\right.$ and ${ }^{119} \mathrm{Sn}$ ) NMR, X-ray and ${ }^{119} \mathrm{Sn}$ Mössbauer spectral studies. Mass fragmentation, thermal decomposition and antimicrobial activities of the complexes are also discussed.
\end{abstract}

\title{
1.1. INTRODUCTION
}

Schiff bases still occupy an important position as ligands in metal coordination chemistry even after almost a century since their discovery. Due to the ease of their preparation, diverse properties, medicinal, biochemical and industrial applications, the keen interest in the study of these compounds arose in the recent years. A number of metal coordination compounds of Schiff bases have been suggested as models to describe the energy transfer in biological systems (1). Formation of the complexes of organotin halides with $\beta$-ketoamines and Schiff bases have recently been of considerable interest amongst various group of chemists to study their structural properties and in view of their potential use as biocides. Sulphur, nitrogen, oxygen and fluorine have long been used to increase the biological activity of an organic moiety (2) and organotin compounds have also found applications in medicinal chemistry $(3,4)$. The Schiff base complexes of organotin(IV) moieties have been widely investigated and the subject has also been reviewed (5). Interest in these compounds may be ascribed to their novel structural features, which are possibly caused by the multidenticity of Schiff base ligands. In view of the rapid progress including salient observations made in the relevant tin(IV) chemistry, an attempt is being made in this article to present a brief up to date review on the organotin(IV) complexes of Schiff bases with special reference to their synthetic methods, structural and thermal behaviour, and biological applications.

\subsection{SYNTHESIS}

\subsubsection{Adducts}

In view of the highly hydrolysable nature of organotin(IV) halides which are generally employed as starting materials, their complexes have been prepared by carrying out the reactions in anhydrous organic solvents such as n-hexane, benzene, acetone, methanol, ethanol or 1,4dioxane 
Tanaka et al $(6,7)$ have synthesized $1: 1$ molecular adducts of dimethyltin(IV) and monon-butyltin(IV) chlorides with several $\mathrm{N}$-substituted pyridine-2-carbaldimines:

$$
\mathrm{R}_{\mathrm{n}} \mathrm{SnCl}_{4-\mathrm{n}}+\mathrm{C}_{5} \mathrm{H}_{4} \mathrm{NCH}=\mathrm{NR}^{\prime} \longrightarrow \mathrm{R}_{\mathrm{n}} \mathrm{SnCl}_{4-\mathrm{n}^{\prime}}\left(\mathrm{C}_{5} \mathrm{H}_{4} \mathrm{NCH}=\mathrm{NR}^{\prime}\right)
$$

$\left[\mathrm{n}=2, \mathrm{R}=\mathrm{Me}, \mathrm{R}^{\prime}=\mathrm{Me}, 4-\mathrm{MeC}_{6} \mathrm{H}_{4}, 4-\mathrm{OCH}_{3} \mathrm{C}_{6} \mathrm{H}_{4}\right.$ and $4-\mathrm{ClC}_{6} \mathrm{H}_{4}(6) ; \mathrm{n}=1, \mathrm{R}=\mathrm{Bu}^{\mathrm{n}}, \mathrm{R}^{\prime}=$ $\mathrm{Me}, \mathrm{Et}, \mathrm{Bu}$, $\mathrm{Ph}, \mathrm{PhCH}_{2}, 4-\mathrm{MeC}_{6} \mathrm{H}_{4}$ and $4-\mathrm{OCH}_{3} \mathrm{C}_{6} \mathrm{H}_{4}$ (7)]

Molecular adducts $(1: 2)$ of mono- and diorganotin(IV) chlorides with various $\mathrm{N}$ alkyl(aryl)salicylaldimines (8-12), N-alkyl(aryl)-2-hydroxyacetophenoneimines (13) and $\mathrm{N}$-alkyl(aryl)2-hydroxy-1-naphthaldimines (14) have been synthesized by mixing the organotin(IV) chlorides with ligands in 1:2 molar ratio in anhydrous $n$-hexane or benzene. The complexes are immediately precipitated on mixing the reactants and only 1:2 adducts are formed even when the reactants are taken in $1: 1$ or $1: 4$ molar ratios $(8,13)$ :

$$
\mathrm{R}_{\mathrm{n}} \mathrm{SnCl}_{4-\mathrm{n}}+2 \mathrm{~L} \longrightarrow \mathrm{R}_{\mathrm{n}} \mathrm{SnCl}_{4-\mathrm{n}} \cdot 2 \mathrm{~L}
$$

[when $\mathrm{L}=2-\mathrm{HOC}_{6} \mathrm{H}_{4} \mathrm{CH}=\mathrm{NR}^{\prime}, \mathrm{R}=\mathrm{R}^{\prime}=\mathrm{Et}, \mathrm{Bu}^{\mathrm{n}}$ and $\mathrm{Ph}, \mathrm{n}=\mathrm{l}(9) ; \mathrm{R}=\mathrm{R}^{\prime}=\mathrm{Me}, \mathrm{Et}, \mathrm{Bu}^{\mathrm{n}}$ and $\mathrm{Ph}, \mathrm{n}=2(8-10) ; \mathrm{R}=\mathrm{Ph}, \mathbf{n}=1 ; \mathrm{R}=\mathrm{Me}$ and $\mathrm{Ph}, \mathrm{n}=2 ; \mathrm{R}^{\prime}=\mathrm{Ph}, 4-\mathrm{ClC}_{6} \mathrm{H}_{4}, 4-$ $\mathrm{MeC}_{6} \mathrm{H}_{4}, 4-\mathrm{OCH}_{3} \mathrm{C}_{6} \mathrm{H}_{4}, 4-\mathrm{NO}_{2} \mathrm{C}_{6} \mathrm{H}_{4}$ and $3-\mathrm{NO}_{2} \mathrm{C}_{6} \mathrm{H}_{4}(11,12)$; when $\mathrm{L}=2-\mathrm{HOC}_{6} \mathrm{H}_{4} \mathrm{C}\left(\mathrm{CH}_{3}\right)$ $=\mathrm{NR}^{\prime}, \mathrm{R}=\mathrm{Et}, \mathrm{Bu}^{\mathrm{n}}$ and $\mathrm{Ph}, \mathrm{n}=1 ; \mathrm{R}=\mathrm{Me}, \mathrm{Et}$ and $\mathrm{Ph}, \mathrm{n}=2 ; \mathrm{R}^{\prime}=\mathrm{Pr}^{\mathrm{n}}, \mathrm{Bu}^{\mathrm{n}}$ and $\mathrm{Ph}(13)$ and when $\mathrm{L}=2-\mathrm{HOC}_{10} \mathrm{H}_{6} \mathrm{CH}=\mathrm{NC}_{6} \mathrm{H}_{4} \mathrm{R}^{\prime}, \mathrm{R}=\mathrm{Ph}, \mathrm{n}=1 ; \mathrm{R}=\mathrm{Me}$ and $\mathrm{Ph}, \mathrm{n}=2 ; \mathrm{R}^{\prime}=\mathrm{H}, 4-\mathrm{Cl}, 4-$ $\mathrm{Me}$ and $\left.4-\mathrm{OCH}_{3}(14)\right]$.

Recently, the complexes of the type $\mathrm{R}_{n} \mathrm{SnCl}_{4 \cdot n} \mathrm{~L}$ where $\mathrm{R}=\mathrm{C}_{6} \mathrm{H}_{5}, \mathrm{n}=2 ; \mathrm{R}=\mathrm{CH}_{3}$ and $\mathrm{C}_{6} \mathrm{H}_{5}$, $\mathbf{n}=3$ and the ligands $(\mathrm{L})$ are neutral bidentate such as N-methyl-S-benzyl- $\beta-N$ (R)methylendithiocarbazates (where $\mathrm{R}=2$-furanyl, 2-methoxyphenyl, 4-methoxyphenyl and benzoylphenyl) have been reported from our laboratory (15).

Monofunctional terdentate Schiff bases obtained from the condensation of 2-aminopyridine with salicylaldehyde and 2-hydroxy-1-naphthaldehyde act only as neutral monodentate ligands yielding $1: 2$ adducts with mono- and diorganotin(IV) halides (16):

$$
\mathrm{R}_{\mathrm{n}} \mathrm{SnX} \mathrm{X}_{4-\mathrm{n}}+2 \mathrm{~L} \longrightarrow \mathrm{R}_{\mathrm{n}} \mathrm{SnX}_{4-\mathrm{n}} \cdot 2 \mathrm{~L}
$$

$[\mathrm{R}=\mathrm{Ph}, \mathrm{X}=\mathrm{Cl}, \mathrm{n}=1 ; \mathrm{R}=\mathrm{Ph}, \mathrm{X}=\mathrm{Cl}$ and $\mathrm{Br}, \mathrm{n}=2 ; \mathrm{R}=\mathrm{Me}, \mathrm{X}=\mathrm{Cl}, \mathrm{n}=2 ; \mathrm{L}=2$ $\mathrm{HOC}_{6} \mathrm{H}_{4} \mathrm{CH}=\mathrm{NC}_{5} \mathrm{H}_{4} \mathrm{~N}$ and $2-\mathrm{HOC}_{10} \mathrm{H}_{6} \mathrm{CH}=\mathrm{NC}_{5} \mathrm{H}_{4} \mathrm{~N}$ (16)].

$\mathrm{N}$-Hydroxyalkylsalicylaldimines and $\mathrm{N}$-hydroxyalkylnaphthaldimines derived from the condensation of ethanolamine or substituted ethanolamines though potentially terdentate act similarly as monodentate forming $1: 2$ adducts with organotin(IV) halides $(17,18)$ :

$$
\mathrm{R}_{\mathrm{n}} \mathrm{SnX}_{4-n}+2\left(\mathrm{R}^{\prime} \mathrm{CH}=\mathrm{NR}{ }^{\prime \prime O H}\right) \longrightarrow \mathrm{R}_{\mathrm{n}} \mathrm{SnX}_{4-\mathrm{n}} \cdot 2\left(\mathrm{R}^{\prime} \mathrm{CH}=\mathrm{NR} \mathrm{NH}^{\prime \prime} \mathrm{OH}\right)
$$

$\left[\mathrm{R}=\mathrm{Ph}, \mathbf{n}=2, \mathrm{X}=\mathrm{Cl} ; \mathrm{R}=\mathrm{Ph}, \mathbf{n}=1, \mathrm{X}=\mathrm{Cl} ; \mathrm{R}^{\prime}=2-\mathrm{HOC}_{6} \mathrm{H}_{4}, \mathrm{R}^{\prime \prime}=\left(\mathrm{CH}_{2}\right)_{2}\right.$ (17); $\mathrm{R}=$ $\mathrm{Ph}, \mathbf{n}=2, \mathrm{X}=\mathrm{CI}$ and $\mathrm{Br} ; \mathrm{R}=\mathrm{Ph}, \mathrm{n}=1, \mathrm{X}=\mathrm{Cl} ; \mathrm{R}^{\prime}=2-\mathrm{HOC}_{10} \mathrm{H}_{6}, \mathrm{R}^{\prime \prime}=\left(\mathrm{CH}_{2}\right)_{2}(17) ; \mathrm{R}=$ $\mathrm{Me}$, Et and $\mathrm{Ph}, \mathrm{n}=2, \mathrm{X}=\mathrm{Cl} ; \mathrm{R}^{\prime}=2-\mathrm{HOC}_{6} \mathrm{H}_{4}, \mathrm{R}^{\prime \prime}=\left(\mathrm{CH}_{2}\right)_{2}, \mathrm{CH}(\mathrm{Me}) \mathrm{CH}_{2}$ and $\mathrm{CH}(\mathrm{Et}) \mathrm{CH}_{2}$ (18)].

Equimolar adducts of $\mathrm{RSnCl}_{3}, \mathrm{R}_{2} \mathrm{SnX}$ and $\mathrm{R}_{3} \mathrm{SnCl}$ with potentially bifunctional tetradentate ligands, N,N'-ethylenebis(acetylacetoneimines) $\left[\mathrm{H}_{2}\right.$ acen] and $\mathrm{N}, \mathrm{N}^{\prime}$-propylenebis(acetylacetoneimines) $\left[\mathrm{H}_{2} \mathrm{acpn}\right]$ have been synthesized by Barbieri et al (19.20) by mixing n-hexane, benzene, acetone 
or 1,4-dioxane solutions of the reactants. The adducts are either precipitated immediately or crystallize from the solution on standing:

$$
\mathrm{R}_{\mathrm{n}} \mathrm{SnX} \mathrm{X}_{4 \mathrm{n}}+\mathrm{H}_{2} \text { acen } / \mathrm{H}_{2} \text { acpn } \longrightarrow \mathrm{R}_{\mathrm{n}} \mathrm{SnX}_{4-\mathrm{n}} \cdot\left(\mathrm{H}_{2} \text { acen } / \mathrm{H}_{2} \text { acpn }\right)
$$

[For $\mathrm{H}_{2}$ acen, $\mathrm{R}=\mathrm{Me}, \mathrm{X}=\mathrm{Cl}, \mathrm{n}=1 ; \mathrm{R}=\mathrm{Me}, \mathrm{X}=\mathrm{Cl}$ and $\mathrm{Br}, \mathrm{n}=2 ; \mathrm{R}=\mathrm{Bu}^{\mathrm{n}}$ and $\mathrm{Ph}, \mathrm{X}=$ $\mathrm{Cl}, \mathbf{n}=2 ; \quad \mathrm{R}=\mathrm{Me}, \mathrm{X}=\mathrm{Cl}, \mathbf{n}=3(19,20)$; for $\mathrm{H}_{2}$ acpn, $\mathrm{R}=\mathrm{Me}$ and $\mathrm{Ph}, \mathrm{X}=\mathrm{Cl}, \mathbf{n}=2$ (19)].

Reactions of mono- and diorganotin(IV) chlorides with equimolar amounts of N,N'ethylenebis(salicylaldimines) $\left[\mathrm{H}_{2}\right.$ salen] and $\mathrm{N}, \mathrm{N}$-propylenebis(salicylaldimines) $\left[\mathrm{H}_{2} \mathrm{salpn}\right]$ in anhydrous ethanol result in the formation of $1: 1$ molecular adducts (21-23):

$$
\mathrm{R}_{\mathrm{n}} \mathrm{SnCl}_{4-\mathrm{n}}+\mathrm{H}_{2} \text { salen } / \mathrm{H}_{2} \text { salpn } \longrightarrow \mathrm{R}_{\mathrm{n}} \mathrm{SnCl}_{4 \mathrm{n}}\left(\mathrm{H}_{2} \text { salen } / \mathrm{H}_{2} \text { salpn }\right)
$$

[For $\mathrm{H}_{2}$ salen, $\mathrm{R}=\mathrm{Me}, \mathrm{Et}, \mathrm{Pr}^{\mathrm{n}}, \mathrm{Bu}^{\mathrm{n}}$ and $\mathrm{Ph}, \mathrm{n}=2$ (21-23); $\mathrm{R}=\mathrm{Me}, \mathrm{Oct}^{\mathrm{n}}$ and $\mathrm{Ph}, \mathrm{n}=1$ (22); for $\mathrm{H}_{2}$ salpn, $\mathrm{R}=\mathrm{Me}, \mathrm{Et}, \mathrm{Pr}^{\mathrm{n}}$ and $\mathrm{Bu}^{\mathrm{n}}, \mathrm{n}=2$ (23)].

Equimolar adducts of $\mathrm{Ph}_{2} \mathrm{SnX}_{2}$ (where $\mathrm{X}=\mathrm{Cl}, \mathrm{ClO}_{4}, \mathrm{NO}_{3}$ and $\mathrm{BF}_{4}$ ) with $\mathrm{N}$, N'-bis(3nitrobenzylidene)ethylenediamine have been synthesized by refluxing in dry methanol (24). Mehrotra and co-workers (25) have synthesized 1:1 molecular adducts of mono- and diorganotin(IV) chlorides with $\mathrm{N}, \mathrm{N}^{\prime}$-ethylenebis(2-hydroxyacetophenoneimines) [ $\mathrm{H}_{2}$ aceten] and $\mathrm{N}, \mathrm{N}^{\prime}$ trimethylenebis(2-hydroxyacetophenoneimines) $\left[\mathrm{H}_{2}\right.$ acetdap] by mixing their benzene solutions:

$$
\mathrm{R}_{\mathrm{n}} \mathrm{SnCl}_{4-\mathrm{n}}+\mathrm{H}_{2} \text { aceten / } \mathrm{H}_{2} \text { acetdap } \longrightarrow \mathrm{R}_{\mathrm{n}} \mathrm{SnCl}_{4-\mathrm{n}} \cdot\left(\mathrm{H}_{2} \text { aceten / } \mathrm{H}_{2} \text { acetdap }\right)
$$

$\left[\mathrm{R}=\mathrm{Et}, \mathrm{Bu}^{\mathrm{n}}\right.$ and $\mathrm{Ph}, \mathrm{n}=1 ; \mathrm{R}=\mathrm{Me}, \mathrm{Et}, \mathrm{Bu}^{\mathrm{n}}$ and $\left.\mathrm{Ph}, \mathrm{n}=2(25)\right]$.

Khoo et al have reported 1:1 molar adducts by the reaction of triphenyltin chloride or triphenyltin isothiocynate with $\mathrm{N}$-arylsalicylideneimines and $\mathrm{N}$-ary l-2-hydroxynaphthylideneimines (26). They have also reported 1:1 molecular adducts of $\mathrm{Me}_{2} \mathrm{SnCl}_{2}$ with $\mathrm{N}$-arylsalicylideneimines (27):

$$
\left(\mathrm{C}_{6} \mathrm{H}_{5}\right)_{3} \mathrm{SnX} /\left(\mathrm{CH}_{3}\right)_{2} \mathrm{SnCl}_{2}+\mathrm{L} \longrightarrow\left(\mathrm{C}_{6} \mathrm{H}_{5}\right)_{3} \mathrm{SnX} . \mathrm{L} /\left(\mathrm{CH}_{3}\right)_{2} \mathrm{SnCl}_{2} \cdot \mathrm{L}
$$

$\left[\mathrm{X}=\mathrm{Cl}\right.$ or $\mathrm{NCS} ; \mathrm{L}=2-\mathrm{HOC}_{6} \mathrm{H}_{4} \mathrm{CH}=\mathrm{NC}_{6} \mathrm{H}_{4} \mathrm{R}, \quad 2-\mathrm{HOC}_{6} \mathrm{H}_{3}\left(\mathrm{OCH}_{3}\right) \mathrm{CH}=\mathrm{NC}_{6} \mathrm{H}_{5}$ and 2$\mathrm{HOC}_{10} \mathrm{H}_{6} \mathrm{CH}=\mathrm{NC}_{6} \mathrm{H}_{4} \mathrm{R}$, where $\mathrm{R}=4-\mathrm{CH}_{3}$ and $4-\mathrm{OCH}_{3}(26)$; for $\left(\mathrm{CH}_{3}\right)_{2} \mathrm{SnCl}_{2}, \mathrm{~L}=2$ $\mathrm{HOC}_{6} \mathrm{H}_{4} \mathrm{CH}=\mathrm{NC}_{6} \mathrm{H}_{4} \mathrm{R}$, where, $\mathrm{R}=\mathrm{H}$ or $\left.4-\mathrm{OCH}_{3}(27)\right]$.

Molecular adducts (1:1) of di- and triorganotin chlorides with various Schiff bases obtained by the condensation of semicarbazide or thiosemicarbazide with salicylaldehyde, 2hydroxynaphthaldehyde, 2-methoxybenzaldehyde, 4-methoxybenzaldehyde, furfuraldehyde, 2hydroxyacetophenone, benzylmethyl ketone, benzil, acetophenone, 4-nitrobenzaldehyde, cyclohexanone, 5-chlorosalicylaldehyde and glyoxal have also been reported from our laboratory $(28,29)$ :

$$
\mathrm{R}_{\mathrm{n}} \mathrm{SnCl}_{4-\mathrm{n}}+\mathrm{R}_{1} \mathrm{R}_{2} \mathrm{C}=\mathrm{NNHC}(\mathrm{X}) \mathrm{NH}_{2} \longrightarrow \mathrm{R}_{\mathrm{n}} \mathrm{SnCl}_{4-\mathrm{n}} \cdot\left[\mathrm{R}_{1} \mathrm{R}_{2} \mathrm{C}=\mathrm{NNHC}(\mathrm{X}) \mathrm{NH}_{2}\right]
$$

$\left[\mathrm{R}=\mathrm{Ph}, \mathrm{n}=3 ; \mathrm{R}_{1}=\mathrm{H}, \quad \mathrm{R}_{2}=2-\mathrm{HOC}_{6} \mathrm{H}_{4}, 4-\mathrm{OCH}_{3} \mathrm{C}_{6} \mathrm{H}_{4}, 2-\mathrm{OCH}_{3} \mathrm{C}_{6} \mathrm{H}_{4}, 2-\mathrm{HOC}_{10} \mathrm{H}_{6}, \mathrm{C}_{4} \mathrm{H}_{3} \mathrm{O}\right.$; $\mathrm{R}_{1}=\mathrm{CH}_{3}, \mathrm{R}_{2}=2-\mathrm{HOC}_{6} \mathrm{H}_{4}, \mathrm{C}_{6} \mathrm{H}_{5} \mathrm{CH}_{2} ; \mathrm{R}_{1}=\mathrm{C}_{6} \mathrm{H}_{5}, \mathrm{R}_{2}=\mathrm{C}_{6} \mathrm{H}_{5} \mathrm{CO} ; \mathrm{X}=\mathrm{O}$ or $\mathrm{S}(28) ; \mathrm{R}_{1}=$ $\mathrm{H}, \mathrm{R}_{2}=\mathrm{H}_{2} \mathrm{NC}(\mathrm{S}) \mathrm{NHN}(\mathrm{C}) \mathrm{H}, 4-\mathrm{NO}_{2} \mathrm{C}_{6} \mathrm{H}_{4} ; \mathrm{R}_{1}=\mathrm{CH}_{3}, \mathrm{R}_{2}=\mathrm{C}_{6} \mathrm{H}_{5} ; \underbrace{\mathrm{R}_{1} \mathrm{R}_{2} \mathrm{C}=\mathrm{C}_{6} \mathrm{H}_{10}} ; \mathrm{X}=\mathrm{S}$ (29) ; $\mathrm{R}=\mathrm{Me}, \mathrm{n}=3, \mathrm{R}_{1}=\mathrm{CH}_{3}, \mathrm{R}_{2}=\mathrm{C}_{6} \mathrm{H}_{5} ; \mathrm{R}_{1}=\mathrm{H}, \mathrm{R}_{2}=4-\mathrm{NO}_{2} \mathrm{C}_{6} \mathrm{H}_{4}, 5-\mathrm{Cl}-2-\mathrm{HOC}_{6} \mathrm{H}_{3}$, $\mathrm{H}_{2} \mathrm{NC}(\mathrm{S}) \mathrm{NHN}(\mathrm{C}) \mathrm{H}, \mathrm{X}=\mathrm{S}(29) ; \mathrm{R}=\mathrm{Ph}, \mathbf{n}=2, \mathrm{R}_{1}=\mathrm{H}, \mathrm{R}_{2}=2-\mathrm{HOC}_{6} \mathrm{H}_{4}, 4-\mathrm{NO}_{2} \mathrm{C}_{6} \mathrm{H}_{4}, 5-\mathrm{Cl}-$ $2-\mathrm{HOC}_{6} \mathrm{H}_{3}, \mathrm{H}_{2} \mathrm{NC}(\mathrm{S}) \mathrm{NHN}(\mathrm{C}) \mathrm{H}, 2-\mathrm{OHC}_{10} \mathrm{H}_{6}, 2-\mathrm{OCH}_{3} \mathrm{C}_{6} \mathrm{H}_{4}, 4-\mathrm{OCH}_{3} \mathrm{C}_{6} \mathrm{H}_{4}, \mathrm{C}_{4} \mathrm{H}_{3} \mathrm{O} ; \mathrm{R}_{1}=\mathrm{CH}_{3}$, 
$\left.\mathrm{R}_{2}=\mathrm{C}_{6} \mathrm{H}_{5}, \mathrm{C}_{6} \mathrm{H}_{5} \mathrm{CH}_{2}, \mathrm{R}_{1} \mathrm{R}_{2} \mathrm{C}=\mathrm{C}_{6} \mathrm{H}_{10} ; \mathrm{X}=\mathrm{S}(29)\right]$.

Nickel(II) and copper(II) chelates of N,N'-ethylenebis(salicylaldimine) [Msalen] and N,N'-ophenylenebis(salicylaldimine) [Msalphen-o] act as bidentate neutral donor ligands and yield 1:1 adducts with mono- and diorganotin(IV) chlorides (30-33):

$$
\mathrm{R}_{\mathrm{n}} \mathrm{SnCl}_{4-\mathrm{n}}+\text { Msalen / Msalphen-o } \longrightarrow \mathrm{R}_{\mathrm{n}} \mathrm{SnCl}_{4-\mathrm{n}} \text {. (Msalen / Msalphen-o) }
$$

[For Msalen, $\mathrm{R}=\mathrm{Me}, \mathrm{Bu}^{\mathrm{n}}$ and $\mathrm{Ph}, \mathbf{n}=1, \mathrm{M}=\mathrm{Ni}(\mathrm{II})(30-32) ; \mathrm{R}=\mathrm{Bu}^{\mathrm{n}}$ and $\mathrm{Ph}, \mathrm{n}=1, \mathrm{M}=$ $\mathrm{Cu}$ (II) (32); $\mathrm{R}=\mathrm{Me}$ and $\mathrm{Ph}, \mathrm{n}=2, \mathrm{M}=\mathrm{Ni}$ (II) $(30,31)$; for Msalphen-o, $\mathrm{R}=\mathrm{Bu}^{\mathrm{n}}$ and $\mathrm{Ph}, \mathrm{n}$ $=1, \mathrm{M}=\mathrm{Ni}(\mathrm{II})$ and $\mathrm{Cu}(\mathrm{II})(33)]$.

Recently, organotin(IV) derivatives of Schiff bases derived from the condensation of 2-hydroxy1-naphthaldehyde or salicylaldehyde with substituted anilines in 1:1 or 1:2 molar ratios have been reported (34).

Recently, di- and triorganotin(IV) derivatives of Schiff bases derived from the condensation of heterocyclic amines, viz., 2-amino-4-phenylthiazole and 2-amino-5-(o-anisyl)-1,3,4-thiadiazole with different aldehydes or ketones have also been reported from our laboratory $(35,36)$

$$
\mathrm{R}_{n} \mathrm{SnCl}_{4-\mathrm{n}}+\mathrm{L} \longrightarrow \mathrm{R}_{\mathrm{n}} \mathrm{SnCl}_{4-n} \mathrm{~L}
$$

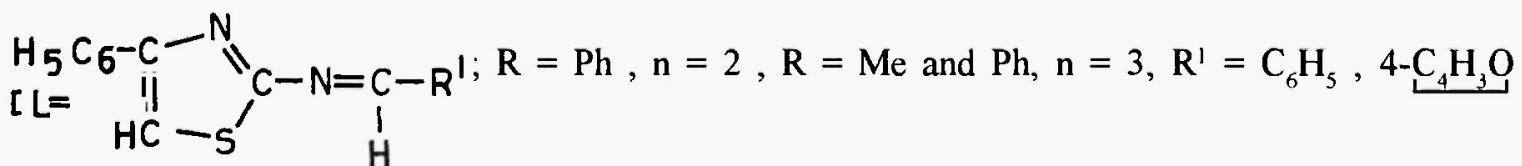

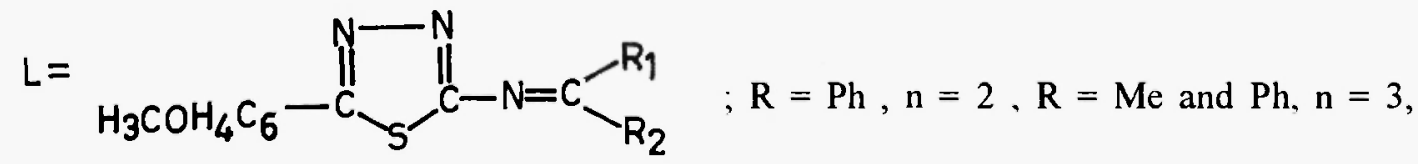

$\mathrm{R}^{1}=\mathrm{H}, \mathrm{R}^{2}=2-\mathrm{HOC}_{6} \mathrm{H}_{4}, 2-\mathrm{HOC}_{10} \mathrm{H}_{6}, \mathrm{C}_{4} \mathrm{H}_{3} \mathrm{O} ; \mathrm{R}^{1}=\mathrm{CH}_{3}, \mathrm{R}^{2}=2-\mathrm{HOC}_{6} \mathrm{H}_{4}, \mathrm{C}_{6} \mathrm{H}_{5} \mathrm{CH}_{2}$, $\left.\mathrm{CH}_{2} \mathrm{COCH}_{3}(36)\right]$

\subsubsection{Substitution Derivatives}

\subsubsection{Deprotonation with triethylamine}

Organotin(IV) chlorides react with dibasic tetradentate Schiff bases in the presence of triethylamine on refluxing in ethanol to yield the desired chelates, $\mathrm{R}_{2} \mathrm{SnL}$ :

$$
\mathrm{R}_{2} \mathrm{SnCl}_{2}+\mathrm{H}_{2} \mathrm{~L} \underset{\mathrm{EtOH}}{\stackrel{2 \mathrm{Et}_{3} \mathrm{~N}}{\longrightarrow}} \mathrm{R}_{2} \mathrm{SnL}+2 \mathrm{Et}_{3} \mathrm{~N} \cdot \mathrm{HCl}
$$

$\left[\mathrm{R}=\mathrm{Me}\right.$ and $\mathrm{Ph} ; \mathrm{H}_{2} \mathrm{~L}=$ Schiff bases derived from ethanolamine and biacetyl/benzil/9:10 phenanthrenequinone, and from ethylenediamine and $o$-vanillin $\left(\mathrm{H}_{2}\right.$ vanilen) $(37) ; \mathrm{R}=\mathrm{Me}, \mathrm{H}_{2} \mathrm{~L}=$ Schiff bases derived from acetoin or benzoin and semicarbazide or thiosemicarbazide (38)].

\subsubsection{Template synthesis}

Reactions of isopropyl amine with diorganotin(IV) monothiodibenzoylmethanates on refluxing in benzene resulted in the formation of diorganotin(IV) chelates of thio- $\beta$-ketoamine (39):

$\mathrm{R}_{2} \mathrm{Sn}[\mathrm{SC}(\mathrm{Ph})=\mathrm{CHCOPh}]_{2}+2 \mathrm{pr}^{\prime} \mathrm{NH}_{2} \rightarrow \mathrm{R}_{2} \mathrm{Sn}\left[\mathrm{SC}(\mathrm{Ph})=\mathrm{CHC}(\mathrm{Ph})=\mathrm{NPr}^{\mathrm{r}}\right]_{2}+2 \mathrm{H}_{2} \mathrm{O}$

[R = Me, Bu" and Ph (39)]. 


\subsubsection{By the sodium salt method}

The sodium salt method has been found to be the most suitable method for the preparation of a wide variety of organotin(IV) chelates of monofunctional bidentate, bifunctional terdentate and tetradentate Schiff bases and $\beta$-ketoamines. Addition of a methanol solution of organotin(IV) halide to a solution of the sodium salt of the Schiff base prepared in situ by the reaction of Schiff base with sodium methoxide in anhydrous methanol under nitrogen results in the formation of the desired chelates:

$$
\mathrm{R}_{2} \mathrm{SnCl}_{2}+\mathrm{H}_{2} \mathrm{~L}+2 \mathrm{NaOMe} \longrightarrow \mathrm{R}_{2} \mathrm{SnL}+2 \mathrm{NaCl}+2 \mathrm{MeOH}
$$

$\left[\mathrm{R}=\mathrm{Me}\right.$ and $\mathrm{Ph}, \quad \mathrm{H}_{2} \mathrm{~L}=2-\mathrm{HOC}_{6} \mathrm{H}_{4} \mathrm{CH}=\mathrm{NC}_{6} \mathrm{H}_{4} \mathrm{OH}-2,2-\mathrm{HOC}_{6} \mathrm{H}_{4} \mathrm{CHNC}_{6} \mathrm{H}_{4} \mathrm{~S}$, $\mathrm{PhCOCH}=\mathrm{C}(\mathrm{Me}) \mathrm{NHC}_{6} \mathrm{H}_{4} \mathrm{OH}-2, \mathrm{MeCOCH}=\mathrm{C}(\mathrm{Me}) \mathrm{NHC}_{6} \mathrm{H}_{4} \mathrm{OH}-2$ and $\mathrm{MeCOCH}_{2} \mathrm{C}(\mathrm{Me}) \mathrm{NHC}_{6} \mathrm{H}_{4} \mathrm{~S}$ (40-44); $\mathrm{R}=\mathrm{Ph}$ and $\mathrm{Bu}, \mathrm{H}_{2} \mathrm{~L}=2-\mathrm{HOC}_{6} \mathrm{H}_{4} \mathrm{CH}=\mathrm{NCH}_{2} \mathrm{CH}_{2} \mathrm{OH}$ (17); $\mathrm{R}=\mathrm{Me}, \mathrm{H}_{2} \mathrm{~L}=$ $\mathrm{HOC}_{6} \mathrm{H}_{3} \mathrm{XCH}=\mathrm{NC}_{6} \mathrm{H}_{3} \mathrm{YOH}-2$ where $\mathrm{X}=\mathrm{H}, \mathrm{Y}=\mathrm{H}, 4-\mathrm{CH}_{3}, 4-\mathrm{Cl}$ and $4-\mathrm{NO}_{2} ; \mathrm{X}=3-\mathrm{OCH}_{3}, \mathrm{Y}$ $=\mathrm{H}, 4-\mathrm{CH}_{3}$ and $4-\mathrm{NO}_{2} ; \mathrm{X}=3-\mathrm{NO}_{2}, \mathrm{Y}=\mathrm{H}, 4-\mathrm{CH}_{3}$ and $4-\mathrm{NO}_{3} ; \mathrm{X}=5-\mathrm{NO}_{2}, \mathrm{Y}=\mathrm{H}, 4-\mathrm{CH}_{3}$ and 4-NO $\mathrm{NO}_{2} ; \mathrm{X}=5-\mathrm{Cl}, \mathrm{Y}=\mathrm{H}$ and 4-Cl (45); $\mathrm{R}=\mathrm{Me}$ and $\mathrm{Bu}^{\prime \prime}, \mathrm{H}_{2} \mathrm{~L}=\mathrm{H}_{2}$ salen (23); $\mathrm{R}=\mathrm{Me}$, Et and $\mathrm{Ph}, \mathrm{H}_{2} \mathrm{~L}=\mathrm{H}_{2}$ salen, $\mathrm{H}_{2}$ salaen, $\mathrm{H}_{2}$ saldap-2-ol and $\mathrm{H}_{2}$ saladap-2-ol $(46,47) ; \mathrm{R}=\mathrm{Me}$ and $\mathrm{Ph}, \mathrm{H}_{2} \mathrm{~L}=\mathrm{H}_{2}$ salen, $\mathrm{H}_{2}$ saldap, $\mathrm{H}_{2}$ salphen-o and $\mathrm{H}_{2}$ vanilen (48)].

Similarly, the chelates of the type $\mathrm{Me}_{2} \mathrm{SnL}$ have been prepared by the reaction of the sodium salts of the Schiff bases derived from the condensation of salicylaldehyde with sulfathiazole, sulfaphenazole, sulfadiazine, sulfaguanidine, 2-( $p$-aminobenzene sulfonamide)-4,5-dimethyloxazole, sulfisoxazole and sulfapyridine with $\mathrm{Me}_{2} \mathrm{SnCl}_{2}(49)$. Reactions of the $\mathrm{Na}$-salts of Schiff bases, $\mathrm{R}^{\prime} \mathrm{C}(\mathrm{OH})=\mathrm{CHC}(\mathrm{Me})=\mathrm{NR}^{2}\left(\mathrm{R}^{1}=\mathrm{Me}, \mathrm{R}^{2}=4-\mathrm{FC}_{6} \mathrm{H}_{4} ; \mathrm{R}^{1}=\mathrm{Ph}, \mathrm{R}^{2}=4-\mathrm{FC}_{6} \mathrm{H}_{4}\right.$ and $\left.2-\mathrm{ClC}_{6} \mathrm{H}_{4}\right)$ with $\mathrm{Me}_{2} \mathrm{SnCl}_{2}$ or $\mathrm{Bu}_{2} \mathrm{SnCl}_{2}$ in $1: 1$ or $1: 2$ molar ratios afford 5- and 6-coordinate tin complexes, $\mathrm{R}_{2} \mathrm{Sn}(\mathrm{Cl}) \mathrm{L}$ or $\mathrm{R}_{2} \mathrm{SnL}_{2}(\mathrm{R}=\mathrm{Me}, \mathrm{Bu} ; \mathrm{L}=$ Schiff base anion) (50). Similarly, 5- or 6-coordinate di- and triorganotin(IV) chelates of semicarbazones and thiosemicarbazones in 1:1 or 1:2 molar ratio have been reported from our laboratory $(51,52)$. Tandon et al have also reported triorganotin(IV) chelates of Schiff bases by the reaction of $\mathrm{Me}_{3} \mathrm{SnCl}$ with the sodium salts of the heterocyclic thiosemicarbazones (53):

$$
\begin{aligned}
& \mathrm{Ph}_{2} \mathrm{SnCl}_{2}+2 \mathrm{NaOMe}+\mathrm{H}_{2} \mathrm{~L} \longrightarrow \mathrm{Ph}_{2} \mathrm{SnL}+2 \mathrm{NaCl}+2 \mathrm{MeOH} \\
& \mathrm{R}_{3} \mathrm{SnCl}+\mathrm{NaOMe}+\mathrm{HL} \longrightarrow \mathrm{R}_{3} \mathrm{SnL}+\mathrm{NaCl}+\mathrm{MeOH} \\
& \mathrm{Ph}_{2} \mathrm{SnCl}_{2}+2 \mathrm{NaOMe}+2 \mathrm{HL}^{\prime} \longrightarrow \mathrm{Ph}_{2} \mathrm{SnL}_{2}^{\prime}+2 \mathrm{NaCl}+2 \mathrm{MeOH}
\end{aligned}
$$

$\left[\mathrm{H}_{2} \mathrm{~L}=\mathrm{R}_{1} \mathrm{R}_{2} \mathrm{C}=\mathrm{NNHC}(\mathrm{X}) \mathrm{NH}_{2} ; \mathrm{X}=\mathrm{O}\right.$ or $\mathrm{S}, \mathrm{R}_{1}=\mathrm{H}, \mathrm{R}_{2}=2-\mathrm{HOC}_{6} \mathrm{H}_{4}, 2-\mathrm{HOC}_{10} \mathrm{H}_{6} ; \mathrm{R}_{1}=$ $\mathrm{CH}_{3}, \mathrm{R}_{2}=2-\mathrm{HOC}_{6} \mathrm{H}_{4},(5 \mathrm{l}) ; \mathrm{X}=\mathrm{S}, \mathrm{R}_{1}=\mathrm{H}, \mathrm{R}_{2}=5-\mathrm{Cl}-2-\mathrm{HOC}_{6} \mathrm{H}_{3}$ (52): $\mathrm{HL}=$ $\mathrm{R}_{1} \mathrm{R}_{2} \mathrm{C}=\mathrm{NNHC}(\mathrm{X}) \mathrm{NH}_{2} ; \mathrm{R}=\mathrm{Ph}, \mathrm{R}_{1}=\mathrm{H}, \mathrm{R},=4-\mathrm{OCH}_{3} \mathrm{C}_{6} \mathrm{H}_{4}, 2-\mathrm{OCH}_{3} \mathrm{C}_{6} \mathrm{H}_{4}, \mathrm{C}_{4} \mathrm{H}_{3} \mathrm{O}: \mathrm{R}_{1}=$ $\mathrm{CH}_{3}, \mathrm{R}_{2}=\mathrm{C}_{6} \mathrm{H}_{5} \mathrm{CH}_{2} ; \mathrm{R}_{1}=\mathrm{C}_{6} \mathrm{H}_{5}, \mathrm{R}_{2}=\mathrm{C}_{6} \mathrm{H}_{5} \mathrm{CO}, \mathrm{X}=\mathrm{O}$ or $\mathrm{S}(5 \mathrm{l}) ; \mathrm{R}=\mathrm{Me}, \mathrm{R}_{1}=\mathrm{CH}_{3}, \mathrm{R}_{2}$ $=\mathrm{C}_{6} \mathrm{H}_{5} ; \mathrm{R}=\mathrm{Ph}, \mathrm{R}_{1}=\mathrm{CH}_{3} \cdot \mathrm{R}_{2}=\mathrm{C}_{6} \mathrm{H}_{5} ; \mathrm{R}_{1}=\mathrm{H}, \mathrm{R}_{2}=4-\mathrm{NO}_{2} \mathrm{C}_{6} \mathrm{H}_{4} ; \mathrm{X}=\mathrm{S}$ (52);HL'= $\left.\mathrm{R}_{1} \mathrm{R}_{2} \mathrm{C}=\mathrm{NNH}(\mathrm{S}) \mathrm{NH}_{2} ; \mathrm{R}_{1}=\mathrm{H}, \mathrm{R}_{2}=4-\mathrm{NO}_{2} \mathrm{C}_{6} \mathrm{H}_{4}(52)\right]$.

A large number of chelates of the types $R_{2} S n L$ and $R_{3} S n L$, where $L=$ anion of Schiff base such as S-alkyl- $\beta$-N-(substituted phenyl)methylendithiocarbazates, have also been reported by the reaction of the sodium salts of the Schiff bases with $\mathrm{R}_{\mathrm{in}} \mathrm{SnCl}_{4-\mathrm{n}}$ (54-58): 


$$
\mathrm{R}_{\mathrm{n}} \mathrm{SnCl}_{4-\mathrm{n}}+\mathrm{Na}_{\mathrm{m}} \mathrm{L} \longrightarrow \mathrm{R}_{\mathrm{n}} \mathrm{SnL}+\mathrm{mNaCl}
$$

$\left[\mathrm{H}_{\mathrm{m}} \mathrm{L}=\mathrm{R}^{1} \mathrm{R}^{2} \mathrm{C}=\mathrm{NN}=\mathrm{C}(\mathrm{SH}) \mathrm{SR}^{3} ; \mathrm{n}=3, \mathrm{~m}=1, \mathrm{R}=\mathrm{C}_{6} \mathrm{H}_{5}, \mathrm{CH}_{3}\right.$ and $\mathrm{C}_{4} \mathrm{H}_{9}, \mathrm{R}^{1}=\mathrm{H}, \mathrm{R}^{2}=2-$ pyridyl, $\mathrm{R}^{3}=\mathrm{CH}_{2} \mathrm{C}_{6} \mathrm{H}_{5}$ and $\mathrm{CH}_{3} ; \mathrm{R}=\mathrm{C}_{6} \mathrm{H}_{5}$ and $\mathrm{C}_{4} \mathrm{H}_{9} . \mathrm{R}^{1}=\mathrm{R}^{3}=\mathrm{CH}_{3}, \mathrm{R}^{2}=\mathrm{CH}_{3}, \mathrm{C}_{2} \mathrm{H}_{5}$ and $\mathrm{C}_{6} \mathrm{H}_{5}$ (54); $\mathrm{n}=2, \mathrm{~m}=2, \mathrm{R}=\mathrm{CH}_{3}, \mathrm{C}_{2} \mathrm{H}_{5}, \mathrm{C}_{4} \mathrm{H}_{9}, \mathrm{C}_{8} \mathrm{H}_{17}$ and $\mathrm{C}_{6} \mathrm{H}_{5} \mathrm{CH}_{2}$ and $\mathrm{n}=3, \mathrm{~m}=$ $1, \mathrm{R}=\mathrm{C}_{6} \mathrm{H}_{5}, \mathrm{R}^{1}=\mathrm{H}, \mathrm{R}^{2}=2-\mathrm{HO}-4-\mathrm{OCH}_{3} \mathrm{C}_{6} \mathrm{H}_{3}, \mathrm{R}^{3}=\mathrm{CH}_{2} \mathrm{C}_{6} \mathrm{H}_{5}(55) ; \mathrm{n}=2, \mathrm{~m}=2, \mathrm{R}=$ $\mathrm{C}_{6} \mathrm{H}_{5}, \mathrm{R}^{1}=\mathrm{H}, \mathrm{R}^{2}=2-\mathrm{HOC}_{6} \mathrm{H}_{4}, 2-\mathrm{HOC}_{10} \mathrm{H}_{6}$ and $\mathrm{R}^{\prime}=\mathrm{CH}_{3}, \mathrm{R}^{2}=2-\mathrm{HOC}_{6} \mathrm{H}_{4} ; \mathrm{n}=3, \mathrm{~m}=1$, $\mathrm{R}=\mathrm{CH}_{3}$ and $\mathrm{C}_{6} \mathrm{H}_{5}, \mathrm{R}^{1}=\mathrm{H}, \mathrm{R}^{2}=2-\mathrm{OCH}_{3} \mathrm{C}_{6} \mathrm{H}_{4}, 4-\mathrm{OCH}_{3} \mathrm{C}_{6} \mathrm{H}_{4}, \mathrm{C}_{4} \mathrm{H}_{3} \mathrm{O}$ and $\mathrm{R}^{1}=\mathrm{C}_{6} \mathrm{H}_{5}, \mathrm{R}^{2}=$ $\mathrm{COC}_{6} \mathrm{H}_{5}, \mathrm{R}^{3}=\mathrm{CH}_{2} \mathrm{C}_{6} \mathrm{H}_{5}(56) ; \mathrm{n}=2, \mathrm{~m}=2, \mathrm{R}=\mathrm{CH}_{2} \mathrm{CH}_{2} \mathrm{CO}_{2} \mathrm{Me}, \mathrm{CH}_{2} \mathrm{CH}_{2} \mathrm{CO}_{2} \mathrm{Et}$ and $\mathrm{CH}_{2} \mathrm{CH}_{2} \mathrm{CO}, \mathrm{Bu}, \mathrm{R}^{1}=\mathrm{H}, \mathrm{R}^{2}=2-\mathrm{HOC}_{6} \mathrm{H}_{4}, 2-\mathrm{HO}-4-\mathrm{OCH}_{3} \mathrm{C}_{6} \mathrm{H}_{3}, 2-\mathrm{HOC}_{10} \mathrm{H}_{6}, \mathrm{R}^{3}=\mathrm{CH}_{3}$ and $\mathrm{C}_{6} \mathrm{H}_{5} \mathrm{CH}_{2}(57,58) ; \mathrm{H}_{\mathrm{m}} \mathrm{L}=\mathrm{R}^{1} \mathrm{R}^{2} \mathrm{C}=\mathrm{NN}\left(\mathrm{CH}_{3}\right) \mathrm{C}=\mathrm{S}\left(\mathrm{SR}^{3}\right), \mathrm{n}=3, \mathrm{~m}=1, \mathrm{R}=\mathrm{CH}_{3}$ and $\mathrm{C}_{6} \mathrm{H}_{5}, \mathrm{R}^{1}$ $=\mathrm{CH}_{3}, \mathrm{R}^{2}=2-\mathrm{HOC}_{6} \mathrm{H}_{4}$ and $\mathrm{R}^{1}=\mathrm{H}, \mathrm{R}^{2}=2-\mathrm{HOC}_{6} \mathrm{H}_{4}, 2-\mathrm{HOC}_{10} \mathrm{H}_{6}, \mathrm{R}^{3}=\mathrm{CH}_{2} \mathrm{C}_{6} \mathrm{H}_{5}$ (15)].

The interaction of the sodium salts of the quadridentate Schiff bases with $\mathrm{R}_{2} \mathrm{SnCl}_{2}(\mathrm{R}=\mathrm{Me}$ and $\mathrm{Ph}$ ) afforded many new organotin(IV) derivatives (59):

$$
\mathrm{R}_{2} \mathrm{SnCl}_{2}+\mathrm{H}_{2} \mathrm{~L}+2 \mathrm{NaOMe} \longrightarrow \mathrm{R}_{2} \mathrm{SnL}+2 \mathrm{NaCl}+2 \mathrm{MeOH}
$$

$\left[H, L=R^{\prime} R^{2} C=N\left(R^{3}\right) N=C^{2} R^{1}\right.$; for $R=\mathrm{CH}_{3}, R^{1}=H, R^{2}=2-H_{0 C} H_{4}, R^{3}=-C_{2}-C_{2}-$, $-\mathrm{CH}_{2} \mathrm{CH}(\mathrm{OH}) \mathrm{CH}_{2}-,-\mathrm{CH}_{2} \mathrm{CH}_{2} \mathrm{CH}_{2}-; \mathrm{R}^{\prime}=\mathrm{CH}_{3}, \mathrm{R}^{2}=2-\mathrm{HOC}_{6} \mathrm{H}_{4}, \mathrm{R}^{3}=-\mathrm{CH}_{2} \mathrm{CH}_{2}-; \mathrm{R}^{1}$ $=\mathrm{H}, \mathrm{R}^{2}=2-\mathrm{HO}-3-\mathrm{COOHC}_{6} \mathrm{H}_{3}, \mathrm{R}^{3}=-\mathrm{CH}_{2}-\mathrm{CH}_{2}-$; for $\mathrm{R}=\mathrm{C}_{6} \mathrm{H}_{5}, \mathrm{R}^{1}=\mathrm{H}, \mathrm{R}^{2}=2-\mathrm{HOC}_{6} \mathrm{H}_{4}$, $\mathrm{R}^{3}=-\mathrm{CH}_{2} \mathrm{CH}_{2}-; \mathrm{R}^{1}=\mathrm{H}, \mathrm{R}^{2}=2-\mathrm{HO}-3-\mathrm{COOHC}_{6} \mathrm{H}_{3}, \mathrm{R}^{3}=-\mathrm{CH}_{2} \mathrm{CH}(\mathrm{OH}) \mathrm{CH}_{2}-$ (59)].

Mehrotra and co-workers (60) have synthesized volatile trialkyltin(IV) complexes of N-substituted salicylaldimines by the reactions of trialkyltin(IV) chlorides with the sodium salts of the Schiff bases prepared in situ from sodium isopropoxide and the Schiff bases in isopropanol:

$$
\mathrm{R}_{3} \mathrm{SnCl}+\mathrm{HOC}_{6} \mathrm{H}_{4} \mathrm{CH}=\mathrm{NR}^{1}+\mathrm{NaOPr}^{\prime} \rightarrow \mathrm{R}_{3} \mathrm{SnOC}_{6} \mathrm{H}_{4} \mathrm{CH}=\mathrm{NR}^{1}+\mathrm{Pr}^{\prime} \mathrm{OH}+\mathrm{NaCl}
$$

$\left[R=E t, R^{\prime}=\mathrm{Me}, \mathrm{Et}\right.$, Allyl, $\mathrm{Pr}^{\mathrm{i}}, \mathrm{Bu}^{\mathrm{n}}$ and $\mathrm{Ph} ; \mathrm{R}=\mathrm{Pr}^{\mathrm{n}}, \mathrm{R}^{1}=\mathrm{Ph} ; \mathrm{R}=\mathrm{Bu}^{\mathrm{n}}, \mathrm{R}^{1}=\mathrm{Bu}^{\mathrm{n}}$ and $\mathrm{Ph}$ (60)].

Recently, some new di- and triorganotin(IV) chelates of Schiff bases derived from the condensation of 2-amino-4-phenylthiazole and 2-amino-5-(o-anisyl)-1,3,4-thiadiazole with different aldehydes or ketones have been reported from our laboratory $(35,36)$.

$$
\mathrm{Ph}_{2} \mathrm{SnCl}_{2} / \mathrm{R}_{3} \mathrm{SnCl}+\mathrm{L}^{\prime} \mathrm{Na} \stackrel{1: 2 / 1: 1}{\longrightarrow} \mathrm{Pl}_{2} \mathrm{SnL}_{2}^{\prime} / \mathrm{R}_{3} \mathrm{SnL}^{\prime}+2 \mathrm{NaCl} / \mathrm{NaCl}
$$

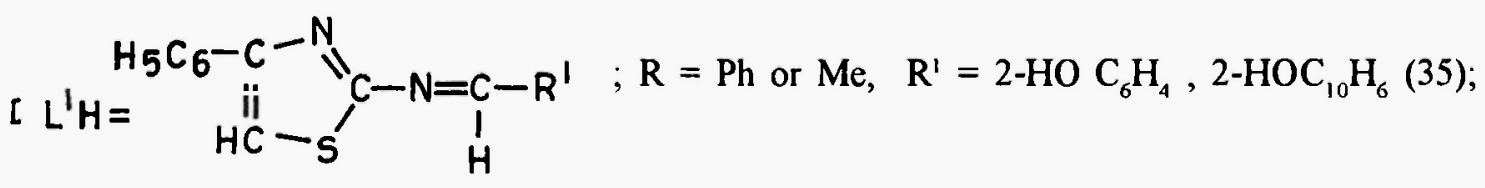<smiles>[R2]C([R2])=Nc1nnc(SCCOC)s1</smiles>
; $\mathrm{R}=\mathrm{Ph}$ or $\mathrm{Me}, \mathrm{R}^{1}=\mathrm{H}, \mathrm{R}^{2}=2-\mathrm{HOC}_{6} \mathrm{H}_{4}$,

$\left.2-\mathrm{HOC}_{10} \mathrm{H}_{6} ; \mathrm{R}^{\prime}=\mathrm{CH}_{3}, \mathrm{R}^{2}=2-\mathrm{HOC}_{6} \mathrm{H}_{4}(36)\right]$.

\subsubsection{By direct $\mathrm{Sn}-\mathrm{Cl}$ bond cleavage reaction using silylated compounds}

Recently, a new series of organotin(IV) compounds have been synthesized by the interaction 
of silylated dibasic quadridentate Schiff bases with $\mathrm{R}_{2} \mathrm{SnCl}_{2}(\mathrm{R}=\mathrm{Me}$ or $\mathrm{Ph})$ and $\mathrm{MeSnCl}_{3}(59$, 61):

$$
\begin{aligned}
& \mathrm{MeSnCl}_{3}+\mathrm{L}\left(\mathrm{SiMe}_{3}\right)_{2} \longrightarrow \mathrm{MeSn}(\mathrm{L}) \mathrm{Cl}+2 \mathrm{Me}_{3} \mathrm{SiCl} \\
& \mathrm{R}_{2} \mathrm{SnCl}_{2}+\mathrm{L}^{\prime}\left(\mathrm{SiMe}_{3}\right)_{2} \longrightarrow \mathrm{R}_{2} \mathrm{SnL}^{\prime}+2 \mathrm{Me}_{3} \mathrm{SiCl}
\end{aligned}
$$

$\left[R=\mathrm{Me}, \mathrm{H}_{2} \mathrm{~L}\right.$ and $\mathrm{H}_{2} \mathrm{~L}^{\prime}=\mathrm{R}^{\prime} \mathrm{R}^{2} \mathrm{C}=\mathrm{N}\left(\mathrm{R}^{3}\right) \mathrm{N}=\mathrm{CR}^{2} \mathrm{R}^{\prime}, \quad \mathrm{R}^{\prime}=\mathrm{CH}_{3}, \mathrm{R}^{2}=2-\mathrm{HOC}_{6} \mathrm{H}_{4}, \mathrm{R}^{3}=-$ $\mathrm{CH}_{2}-\mathrm{CH}_{2}-$ and $\mathrm{C}_{6} \mathrm{H}_{4}(61) ; \mathrm{R}=\mathrm{Me}$ or $\mathrm{Ph}, \mathrm{H}_{2} \mathrm{~L}^{\prime}=$ Schiff bases (59), as indicated in Eq. (19)]. When mononuclear complexes $\left[\mathrm{H}_{2}\right.$ salcen] $\mathrm{SnR}_{2}$, where $\left[\mathrm{H}_{2}\right.$ salcen $=\mathrm{N}, \mathrm{N}$-ethylenebis(3-carboxysalicylaldimine), $\mathrm{R}=\mathrm{Me}$ or $\mathrm{Ph}$ ] react with trimethylsilyl-N-N'-diphenylurea (TDPU) in THF resulting the silylated complexes $\left[\left(\mathrm{Me}_{3} \mathrm{Si}\right)_{2}\right.$ salcenSnR $\mathrm{R}_{2}$. Desilylation of mononuclear complexes with $\mathrm{R}_{2} \mathrm{SnCl}_{2}(\mathrm{R}=\mathrm{Me}$ or $\mathrm{Ph})$ yields di-tin $(\mathrm{Sn}-\mathrm{Sn})$ complexes $\left[\mathrm{R}_{2} \mathrm{Sn}(\right.$ salcen$\left.) \mathrm{SnR}_{2}\right]$. On the other hand, the silylated products of the mononuclear metal complexes $\left[\mathrm{M}\left(\mathrm{H}_{2}\right.\right.$ salcen $\left.)\right](\mathrm{M}=\mathrm{Ni}$ or $\mathrm{Pd})$ react with $\mathrm{R}_{2} \mathrm{SnCl}_{2}$ to yield hetero dinuclear complexes [M(salcen)SnR $\left.\mathrm{S}_{2}\right]$ with $\mathrm{Ni}-\mathrm{Sn}$ and $\mathrm{Pd}-\mathrm{Sn}$ bonds. $\underset{\text { Mononuclear complex }}{\left[\mathrm{H}_{2} \text { salcen } \mathrm{SnR}_{2}\right.} \underset{1: 2}{\longrightarrow}\left[\left(\mathrm{Me}_{3} \mathrm{Si}\right)_{2} \mathrm{salcenSnR}_{2}\right] \stackrel{\mathrm{R}_{2} \mathrm{SnCl}_{2}}{\longrightarrow} \underset{\begin{array}{c}\text { TDPU in } \mathrm{THF} \\ \text { dinuclear complex }\end{array}}{\left.\left[\mathrm{R}_{2} \mathrm{Sn}(\text { salcen }) \mathrm{SnR}_{2}\right)\right]}$

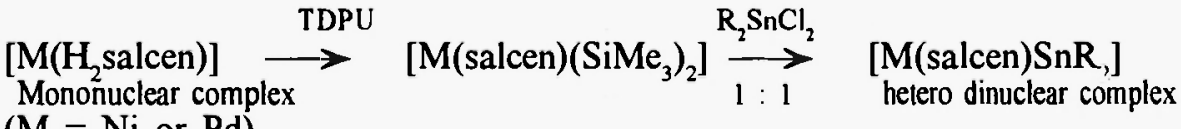

$$
\begin{aligned}
& (\mathrm{M}=\mathrm{Ni} \text { or } \mathrm{Pd})
\end{aligned}
$$

The reactions of tetraorganosilicon compounds such as $\mathrm{RCH}_{2} \mathrm{SiMe}_{3}$ with $\mathrm{LSnCl}_{2}$ where $\mathrm{L}=$ anion of dibasic quadridentate Schiff bases have been reported to yield new organotin(IV) compounds (62):

$$
\begin{gathered}
\mathrm{LSnCl}_{2}+2 \mathrm{C}_{6} \mathrm{H}_{5} \mathrm{CH}_{2} \mathrm{SiMe}_{3} \longrightarrow \mathrm{C}_{6} \mathrm{H}_{5} \mathrm{CH}_{2}(\mathrm{LSn}) \mathrm{CH}_{2} \mathrm{C}_{6} \mathrm{H}_{5}+2 \mathrm{Me}_{3} \mathrm{SiCl} \\
\mathrm{nLSnCl}_{2}+\mathrm{nMe}_{3} \mathrm{SiCH}_{2} \mathrm{C}_{6} \mathrm{H}_{4} \mathrm{CH}_{2} \mathrm{SiMe}_{3} \longrightarrow \\
{\left[-\mathrm{Sn}_{2}-\mathrm{CH}_{2} \mathrm{C}_{6} \mathrm{H}_{4} \mathrm{CH}_{2}(\mathrm{LSn}) \mathrm{CH}_{2} \mathrm{C}_{2} \mathrm{H}_{2} \mathrm{CH}_{2}-\mathrm{Sn}^{\prime} \mathrm{n}-\right]+2 \mathrm{nMer}_{3} \mathrm{SiCl}}
\end{gathered}
$$

\subsubsection{By the potassium salt method}

Recently, few di- and triorganotin(IV) derivatives of the Schiff base derived from $o$ hydroxyacetophenone and glycine have been synthesized by the reaction of the corresponding diand triorganotin(IV) chlorides with the potassium salt of the Schiff base in 1:2 and 1:1 molar ratios, respectively, in refluxing benzene (63):

$$
\begin{array}{r}
\mathrm{R}_{\mathrm{n}} \mathrm{SnCl}_{4 \mathrm{n}}+\mathrm{m}\left[\mathrm{HOC}_{6} \mathrm{H}_{4} \mathrm{C}(\mathrm{Me})=\mathrm{NCH}_{2} \mathrm{COOK}\right] \longrightarrow \\
{\left[\mathrm{HOC}_{6} \mathrm{H}_{4} \mathrm{C}(\mathrm{Me})=\mathrm{NCH}_{2} \mathrm{COO}\right]_{m} \mathrm{SnR}_{\mathrm{n}}+\mathrm{mKCl}}
\end{array}
$$

$[\mathrm{n}=3, \mathrm{~m}=1 ; \mathrm{n}=2, \mathrm{~m}=2 ; \mathrm{R}=\mathrm{Me}, \mathrm{Bu}$ and $\mathrm{Ph}(63)]$.

However, a reaction between diorganotin(IV) dichloride and the potassium salt of the Schiff base in refluxing benzene in the presence of triethylamine yielded the corresponding 5-coordinate diorganotin(IV) derivatives (63):

$$
\begin{array}{r}
\mathrm{R}_{2} \mathrm{SnCl}_{2}+\left[\mathrm{HOC}_{6} \mathrm{H}_{4} \mathrm{C}(\mathrm{Me})=\mathrm{NCH}_{2} \mathrm{COOK}\right] \stackrel{\mathrm{Et}_{3} \mathrm{~N}}{\longrightarrow} \\
{\left[\mathrm{OC}_{6} \mathrm{H}_{4} \mathrm{C}(\mathrm{Me})=\mathrm{NCH}_{2} \mathrm{COO}\right] \mathrm{SnR}_{2}+\mathrm{KCl}+\mathrm{Et}_{3} \mathrm{~N} \cdot \mathrm{HCl}}
\end{array}
$$

$[\mathrm{R}=\mathrm{Me}, \mathrm{Bu}$ and $\mathrm{Ph}]$ 
Recently, 5-coordinate organotin chelates have been prepared by treating 2$\mathrm{HOC}_{6} \mathrm{H}_{4} \mathrm{CH}=\mathrm{NCH}(\mathrm{R}) \mathrm{CO}_{2} \mathrm{~K}$ with $\mathrm{R}_{2}^{\prime} \mathrm{SnCl}_{2}\left[\mathrm{R}=\mathrm{H}, \mathrm{Me}, \mathrm{Me}_{2} \mathrm{CHCH}_{2}, \mathrm{PhCH}_{2}\right.$ and $\mathrm{MeSCH}_{2} \mathrm{CH}_{2}$; $\mathrm{R}^{\prime}=\mathrm{Bu}$ and $\left.\mathrm{Ph}(64,65)\right]$.

\subsubsection{By the thallium salt method}

N,N'-Ethylenebis(salicylaldiminato)dimethyltin(IV), N,N'-ethylenebis(salicylaldiminato)diphenyltin(IV) and (N-2-hydroxyphenylsalicylaldiminato)dimethyltin(IV) have been synthesized by the reaction of diorganotin(IV) chlorides with the thallium salts of the respective Schiff bases in anhydrous methanol or benzene (21):

$$
\begin{aligned}
&\left.\mathrm{R}_{2} \mathrm{SnCl}_{2}+\mathrm{Tl}_{2} \text { salen } \stackrel{\mathrm{MeOH}}{\longrightarrow} \underset{\mathrm{R}}{\longrightarrow} \mathrm{Me} \text { and } \mathrm{Ph}\right] \text { Bensalen }+2 \mathrm{TlCl} \downarrow \\
& \mathrm{Me}_{2} \mathrm{SnCl}_{2}+\mathrm{Tl}_{2} \text { salap } \longrightarrow \mathrm{Me}_{2} \text { Snsalap }+2 \mathrm{TlCl} \downarrow
\end{aligned}
$$

\subsubsection{By ligand exchange}

Monohalomonoorganotin(IV) chelates of bifunctional terdentate Schiff bases have been synthesized by the reactions of dimethyltin(IV) chelates of the Schiff bases with monoorganotin(IV) halides in $n$-hexane under dry nitrogen (66-68). The chelates either precipitate or crystallize out leaving dimethyltin(IV) dichloride in solution:

$$
\mathrm{RSnCl}_{3}+\mathrm{Me}_{2} \mathrm{SnL} \longrightarrow \mathrm{RSnClL}+\mathrm{Me}_{2} \mathrm{SnCl}_{2}
$$

$\left[\mathrm{R}=\mathrm{Me}, \mathrm{Oct}^{\mathrm{n}}\right.$ and $\mathrm{Ph} ; \mathrm{L}$ is the dianion of $2-\mathrm{HOC}_{6} \mathrm{H}_{4} \mathrm{CH}=\mathrm{NC}_{6} \mathrm{H}_{4} \mathrm{OH}-2, \mathrm{PhC}(\mathrm{OH})=$ $\mathrm{CHC}(\mathrm{Me})=\mathrm{NC}_{6} \mathrm{H}_{4} \mathrm{OH}-2,2-\mathrm{HOC}_{6} \mathrm{H}_{4} \mathrm{CHNHC}_{6} \mathrm{H}_{4} \mathrm{~S}$ or $\mathrm{MeCOCH}_{2} \mathrm{C}(\mathrm{Me}) \mathrm{NHC}_{6} \mathrm{H}_{4} \mathrm{~S}$ (66-68)].

\subsubsection{By the reaction of organotin(IV) amides with Schiff bases}

$\mathrm{N}, \mathrm{N}$ '-Ethylenebis(acetylacetoneiminato)trimethyltin(IV) and -dimethyltin(IV) have been prepared by the reactions of $\mathrm{Me}_{3} \mathrm{SnNEt}_{2}$ and $\mathrm{Me}_{2} \mathrm{Sn}\left(\mathrm{NEt}_{2}\right)_{2}$, respectively with $\mathrm{H}_{2}$ acen in refluxing benzene under dry nitrogen (69):

$$
\begin{aligned}
& \mathrm{Me}_{3} \mathrm{SnNEt}_{2} / \mathrm{Me}_{2} \mathrm{Sn}\left(\mathrm{NEt}_{2}\right)_{2}+\mathrm{H}_{2} \text { acen } \longrightarrow \\
& \mathrm{Me}_{3} \mathrm{SnHacen} / \mathrm{Me}_{2} \text { Snacen }+\mathrm{Et}_{2} \mathrm{NH} / 2 \mathrm{Et}_{2} \mathrm{NH}
\end{aligned}
$$

\subsubsection{By the reaction of trialkyltin(IV) alkoxides with Schiff bases}

Trialkyltin(IV) derivatives of $\mathrm{N}$-alkyl(aryl)salicylaldimines and 2,2-disubstituted benzothiazolines have been prepared by reacting the trialkyltin(IV) alkoxides with the Schiff bases in refluxing benzene $(60,70)$. The alcohol liberated during the course of the reaction is removed azeotropically with benzene for pushing the reactions to completion and the complexes are purified by distillation under reduced pressure. The same method (71) has also been used for the synthesis of dialkyltin(IV) derivatives of salicylaldimines:

$$
\mathrm{R}_{3} \mathrm{SnOR}^{\prime \prime}+\mathrm{HOC}_{6} \mathrm{H}_{4} \mathrm{CH}=\mathrm{NR}^{\prime} \longrightarrow \mathrm{R}_{3} \mathrm{SnOC}_{6} \mathrm{H}_{4} \mathrm{CH}=\mathrm{NR}^{\prime}+\mathrm{R}^{\prime \prime} \mathrm{OH}
$$

$\left[R=\operatorname{Pr}^{n}, R^{\prime}=M e, E t\right.$, Allyl, $\operatorname{Pr}^{i}$ and $B^{\prime \prime}, R^{\prime \prime}=E t ; R=B u^{n}, R^{\prime}=M e, E t$, Allyl and $\operatorname{Pr}^{i}, R^{\prime \prime}=$ $\left.\operatorname{Pr}^{i}(60)\right]$.

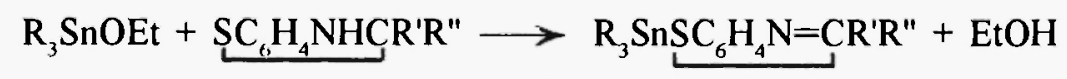


$\left[\mathrm{R}=\mathrm{Et}, \mathrm{Pr}^{\mathrm{n}}\right.$ and $\mathrm{Bu}^{\mathrm{n}} ; \mathrm{R}^{\prime}=\mathrm{Me}, \mathrm{R}^{\prime \prime}=\mathrm{Me}, \mathrm{Et}, \mathrm{Bu}^{\mathrm{i}}$ and $\mathrm{Ph} ; \mathrm{R}^{\prime}=\mathrm{Et}, \mathrm{R}^{\prime \prime}=\mathrm{Et}$ and $\left.\mathrm{Bu}^{\mathrm{n}}(70)\right]$.

$$
\mathrm{R}_{2} \mathrm{Sn}\left(\mathrm{OPr}^{\mathrm{i}}\right)_{2}+\mathrm{HOC}_{6} \mathrm{H}_{4} \mathrm{C}\left(\mathrm{R}^{\prime}\right)=\mathrm{NR} \mathrm{N}^{\prime \prime} \mathrm{OH} \longrightarrow \mathrm{R}_{2} \overline{\mathrm{SnOC}_{6} \mathrm{H}_{4} \mathrm{C}\left(\mathrm{R}^{\prime}\right)=\mathrm{NR}^{\prime \prime}}+2 \mathrm{Pr}^{\prime} \mathrm{OH}
$$

$\left[\mathrm{R}=\mathrm{Me}, \mathrm{Et}, \mathrm{Bu}\right.$ and $\mathrm{Ph}, \mathrm{R}^{\prime}=\mathrm{H}$ and $\mathrm{Me}, \mathrm{R}^{\prime \prime}=-\mathrm{CH}_{2} \mathrm{CH}_{2}-$ and $\left.-\mathrm{CH}_{2} \mathrm{CH}_{2} \mathrm{CH}_{2}-(71)\right]$.

\subsubsection{By the reactions of trialkyltin hydroxides and Schiff bases}

Trimethyltin(IV) hydroxide reacts with 2,2-disubstituted benzothiazolines to yield trimethyltin(IV) chelates. Water formed during the course of the reaction is removed azeotropically with benzene and the chelates are purified by distillation under vacuum (70):

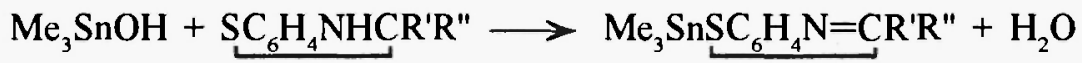

$\left[\mathrm{R}^{\prime}=\mathrm{Me}, \mathrm{R}^{\prime \prime}=\mathrm{Me}, \mathrm{Et}, \mathrm{Bu}^{\mathrm{i}}\right.$ and $\mathrm{Ph} ; \mathrm{R}^{\prime}=\mathrm{Et}, \mathrm{R}^{\prime \prime}=\mathrm{Et}$ and $\left.\mathrm{Bu}^{\mathrm{n}}(70)\right]$.

\subsection{By the reaction of diorganotin(IV) oxides with Schiff bases}

Reactions of diorganotin(IV) oxides with $\mathrm{N}$-isopropylthiodibenzoylmethaneimine [HtdbmPri] in refluxing benzene result in the formation of diorganotin(IV) chelates and the liberation of water which is removed as an azeotrope with benzene (39):

$$
\mathrm{R}_{2} \mathrm{SnO}+2 \mathrm{HtdbmPr}^{\mathrm{C}} \stackrel{\mathrm{C}_{6} \mathrm{H}_{6}}{\longrightarrow} \quad \mathrm{R}_{2} \mathrm{Sn}\left(\mathrm{tdbmPr}^{\mathrm{i}}\right)_{2}+\mathrm{H}_{2} \mathrm{O}
$$

$\left[\mathrm{R}=\mathrm{Me}, \mathrm{Bu}^{\mathrm{n}}\right.$ and $\left.\mathrm{Ph}(39)\right]$.

Similarly, di- and tributyltin(IV) chelates of semicarbazones and thiosemicarbazones have been prepared $(38,52,72,73)$ :

$$
\mathrm{Bu}_{2} \mathrm{SnO}+\mathrm{H}_{2} \mathrm{~L} / 2 \mathrm{HL} \longrightarrow \mathrm{Bu}_{2} \mathrm{SnL} / \mathrm{Bu}_{2} \mathrm{SnL}_{2}+\mathrm{H}_{2} \mathrm{O}
$$

$\left[\mathrm{H}_{2} \mathrm{~L} / \mathrm{HL}=\mathrm{R}^{1} \mathrm{R}^{2} \mathrm{C}=\mathrm{NN}=\mathrm{C}(\mathrm{XH}) \mathrm{NH}_{2}, \mathrm{R}^{1}=\mathrm{H}, \mathrm{R}^{2}=2-\mathrm{HOC}_{6} \mathrm{H}_{4}, \mathrm{X}=\mathrm{S}(72)\right.$ and $\mathrm{X}=\mathrm{O}$ and $S(73) ; R^{1}=H, R^{2}=2-H_{10} H_{6}, X=S ; R^{1}=H, R^{2}=C_{6} H_{5}$ and $R^{1}=C_{3}, R^{2}=2-$ $\mathrm{HOC}_{6} \mathrm{H}_{4}, \mathrm{X}=\mathrm{O}$ and $\mathrm{S}(73) ; \mathrm{R}^{1}=\mathrm{CH}_{3}, \mathrm{R}^{2}=\mathrm{HC}\left(\mathrm{CH}_{3}\right) \mathrm{OH}$ and $\mathrm{R}^{1}=\mathrm{C}_{6} \mathrm{H}_{5}, \mathrm{R}^{2}=$ $\mathrm{HC}\left(\mathrm{C}_{6} \mathrm{H}_{5}\right) \mathrm{OH}, \mathrm{X}=\mathrm{O}$ and $\mathrm{S}(38) ; \mathrm{R}^{\prime}=\mathrm{H}, \mathrm{R}^{2}=5-\mathrm{Cl}-2-\mathrm{OHC}_{6} \mathrm{H}_{3} ; \mathrm{R}^{1}=\mathrm{CH}_{3}, \mathrm{R}^{2}=\mathrm{C}_{6} \mathrm{H}_{5}$; $\left.\underline{\mathrm{R}^{\prime} \mathrm{R}^{2} \mathrm{C}}=\underline{\mathrm{C}_{6} \mathrm{H}_{10}} ; \mathrm{X}=\mathrm{S}(52)\right]$.

$$
\left(\mathrm{Bu}_{3} \mathrm{Sn}\right)_{2} \mathrm{O}+2 \mathrm{HL} \longrightarrow 2 \mathrm{Bu}_{3} \mathrm{SnL}+\mathrm{H}_{2} \mathrm{O}
$$

$\left[\mathrm{HL}=\mathrm{R}^{1} \mathrm{R}^{2} \mathrm{C}=\mathrm{NN}=\mathrm{C}(\mathrm{XH}) \mathrm{NH}_{2}, \mathrm{R}^{1}=\mathrm{H}, \mathrm{R}^{2}=\mathrm{C}_{6} \mathrm{H}_{5}\right.$ and $\mathrm{R}^{\prime}=\mathrm{CH}_{3}, \mathrm{R}^{2}=2-\mathrm{HOC}_{6} \mathrm{H}_{4} ; \mathrm{X}=\mathrm{O}$ and $S(73)]$.

The same method has also been adopted for the synthesis of a number of penta- and hexacoordinate organotin(IV) chelates of the Schiff bases derived from S-alkyldithiocarbazate amines (72, 74-76):

$\mathrm{R}_{\mathrm{n}} \mathrm{SnO} /\left(\mathrm{R}_{\mathrm{m}} \mathrm{Sn}\right)_{2} \mathrm{O}+\mathrm{H}_{2} \mathrm{~L} \longrightarrow \mathrm{R}_{\mathrm{n}} \mathrm{SnL} /\left(\mathrm{R}_{\mathrm{m}} \mathrm{Sn}\right)_{2} \mathrm{~L}+\mathrm{H}_{2} \mathrm{O}$

$\left[\mathrm{H}_{2} \mathrm{~L}=\mathrm{R}^{1} \mathrm{R}^{2} \mathrm{C}=\mathrm{NNC}(\mathrm{SH}) \mathrm{SR}^{3} ; \mathrm{n}=2, \mathrm{R}=\mathrm{Bu}, \mathrm{R}^{1}=\mathrm{H}\right.$ and $\mathrm{CH}_{3}, \mathrm{R}^{2}=2-\mathrm{HOC}_{6} \mathrm{H}_{4}, \mathrm{R}^{3}=\mathrm{CH}_{3}$ (74); $\mathrm{n}=2, \mathrm{R}=\mathrm{Bu}, \mathrm{R}^{1}=\mathrm{H}, \mathrm{R}^{2}=2-\mathrm{HOC}_{6} \mathrm{H}_{4}, \mathrm{R}^{3}=\mathrm{CH}_{2} \mathrm{C}_{6} \mathrm{H}_{5}(72,76) ; \mathrm{n}=2, \mathrm{R}=\mathrm{Me}, \mathrm{Bu}$ and $\mathrm{Ph}, \mathrm{R}^{1}=\mathrm{CH}_{3}, \mathrm{R}^{2}=2-\mathrm{HOC}_{6} \mathrm{H}_{4} ; \mathrm{n}=2, \mathrm{R}=\mathrm{Me}, \mathrm{Bu}$ and $\mathrm{Oct}$ and $\mathrm{m}=3, \mathrm{R}=\mathrm{Bu}$ and $\mathrm{Ph}, \mathrm{R}^{1}=\mathrm{H}, \mathrm{R}^{2}=2-\mathrm{HO}-4-\mathrm{ClC}_{6} \mathrm{H}_{3}$ and 2-HO-4- $\mathrm{BrC}_{6} \mathrm{H}_{3} ; \mathrm{R}^{3}=\mathrm{CH}_{2} \mathrm{C}_{6} \mathrm{H}_{5}(75) ; \mathrm{n}=2, \mathrm{R}=\mathrm{Me}$, $\mathrm{Bu}$ and Oct and $\mathrm{m}=3, \mathrm{R}=\mathrm{Bu}$, Oct and $\left.\mathrm{Ph}, \mathrm{R}^{1}=\mathrm{H}, \mathrm{R}^{2}=2-\mathrm{HOC}_{6} \mathrm{H}_{4}, \mathrm{R}^{3}=\mathrm{CH}_{2} \mathrm{C}_{6} \mathrm{H}_{5}(76)\right]$.

Recently, some new dibutyltin(IV) chelates of the Schiff bases derived from the condensation of 2-amino-5-(o-anisyl)-1,3,4-thiadiazole with salicylaldehyde, 2-hydroxynaphthaldehyde and 2- 
hydroxyactophenone have been prepared from our laboratory (36).

$$
\left[L^{\prime} H=\underset{\left.\mathrm{H}_{3} \mathrm{COH}_{4} \mathrm{C}_{6}-\mathrm{C}_{2}=2-\mathrm{HOC}_{6} \mathrm{H}_{4}(36)\right] .}{\mathrm{Bu}_{2} \mathrm{SnO}+2 \mathrm{~L}^{\prime} \mathrm{H}} \stackrel{\mathrm{N}=\mathrm{C}_{\mathrm{R}}^{\mathrm{N}}}{-\mathrm{R}_{1}} ; \mathrm{R}_{1}=\mathrm{H}, \mathrm{R}\right.
$$

Sandhu et al have also synthesized new organotin(IV) chelates by the reaction of $\mathrm{R}_{2} \mathrm{SnO}$ and the Schiff bases derived from the condensation of aromatic aldehydes with $o$-arsanilic acid (1:1) and from ethylenediamine with acetylacetone (1:2) (77):

$$
\begin{aligned}
& \mathrm{R}_{2} \mathrm{SnO}+\mathrm{H}_{2} \mathrm{~L} \longrightarrow \mathrm{R}_{2} \mathrm{SnL}+\mathrm{H}_{2} \mathrm{O} \\
& \mathrm{R}_{2} \mathrm{SnO}+\mathrm{H}_{2} \mathrm{SB}^{\prime} \longrightarrow \mathrm{R}_{2} \mathrm{SnSB}^{\prime} / \mathrm{R}_{2} \mathrm{Sn}\left(\mathrm{HSB}^{\prime}\right)_{2}+\mathrm{H}_{2} \mathrm{O}
\end{aligned}
$$

$\left[\mathrm{R}=\mathrm{Me}, \mathrm{Bu}\right.$, Oct and Benz, $\mathrm{H}_{2} \mathrm{~L}=\mathrm{R}^{1} \mathrm{R}^{2} \mathrm{C}=\mathrm{NC}_{6} \mathrm{H}_{4} \mathrm{AsO}_{3} \mathrm{H}_{2}, \mathrm{R}^{1}=\mathrm{H}, \mathrm{R}^{2}=\mathrm{C}_{6} \mathrm{H}_{5}, 2-\mathrm{NO}_{2} \mathrm{C}_{6} \mathrm{H}_{4}$, $2-\mathrm{ClC}_{6} \mathrm{H}_{4}, 3-\mathrm{NO}_{2} \mathrm{C}_{6} \mathrm{H}_{4} ; \mathrm{H}_{2} \mathrm{SB}^{\prime}=\left[-\mathrm{CH}_{2} \mathrm{~N}=\mathrm{C}\left(\mathrm{CH}_{3}\right) \mathrm{CH}=\mathrm{C}(\mathrm{OH}) \mathrm{CH}_{3}\right]_{2}$ (77) $]$.

Similarly, the reactions of diorganotin(IV) oxides and bis(tri-n-butyltin) oxide with the azomethines derived by the condensation of salicylaldehyde with various sulfadrugs, by the condensation of salicylaldehyde, $o$-hydroxyacetophenone or benzaldehyde with $o$-aminothiophenol, and by salicylaldehyde or $o$-hydroxynaphthaldehyde with $p$-flouroaniline yield $1: 1$ organotin(IV) chelates (78, 79):

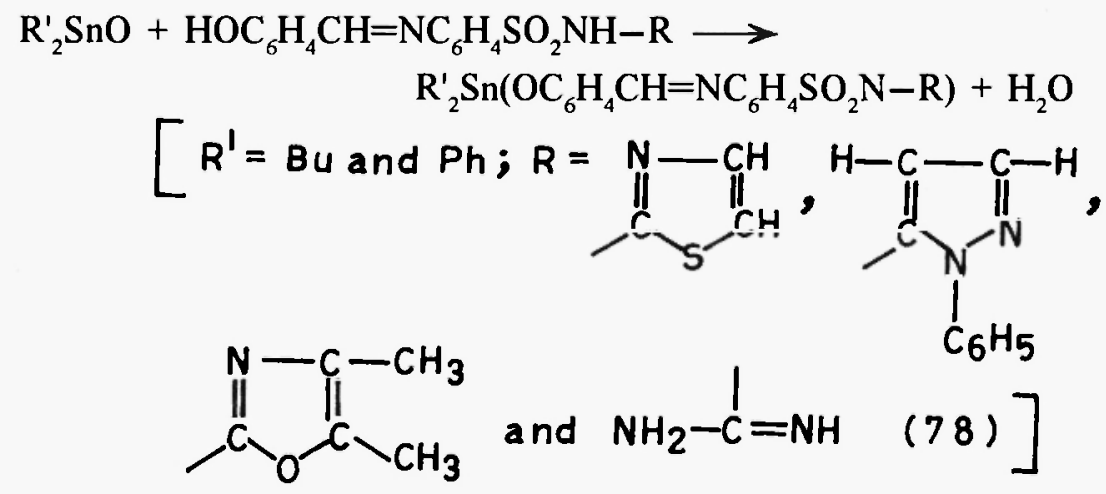

$$
\begin{aligned}
& \mathrm{Bu}_{2} \mathrm{SnO}+\mathrm{H}_{2} \mathrm{~L} / 2 \mathrm{HL}^{\prime} \longrightarrow \mathrm{Bu}_{2} \mathrm{SnL} / \mathrm{Bu}_{2} \mathrm{SnL}_{2}^{\prime}+\mathrm{H}_{2} \mathrm{O} \\
& \left(\mathrm{Bu}_{3} \mathrm{Sn}\right)_{2} \mathrm{O}+2 \mathrm{HL}^{\prime} \longrightarrow 2 \mathrm{Bu}_{3} \mathrm{SnL}^{\prime}+\mathrm{H}_{2} \mathrm{O}
\end{aligned}
$$

$\left[\mathrm{H}_{2} \mathrm{~L}=2-\mathrm{HOC}_{6} \mathrm{H}_{4} \mathrm{C}\left(\mathrm{CH}_{3}\right)=\mathrm{NC}_{6} \mathrm{H}_{4} \mathrm{SH}\right.$ and $2-\mathrm{HOC}_{6} \mathrm{H}_{4} \mathrm{CH}=\mathrm{NC}_{6} \mathrm{H}_{4} \mathrm{SH} ; \mathrm{HL}=2-\mathrm{HOC}_{6} \mathrm{H}_{4} \mathrm{CH}$ $=\mathrm{NC}_{6} \mathrm{H}_{4} \mathrm{~F}-4,2-\mathrm{HOC}_{10} \mathrm{H}_{6} \mathrm{CH}=\mathrm{NC}_{6} \mathrm{H}_{4} \mathrm{~F}-4$ and $\left.\mathrm{C}_{6} \mathrm{H}_{5} \mathrm{CH}=\mathrm{NC}_{6} \mathrm{H}_{4} \mathrm{SH}(79)\right]$.

Some new dibutyltin(IV) chelates of the dianionic tridentate Schiff bases derived from the condensation of salicylaldehyde or $o$-hydroxyacetophenone or pyruvic acid with different amino acids have recently been reported from our laboratory (80):

$$
\mathrm{Bu}_{2} \mathrm{SnO}+\mathrm{H}_{2} \mathrm{~L} \stackrel{\text { benzene }}{\longrightarrow} \mathrm{Bu}_{2} \mathrm{SnL}+\mathrm{H}_{2} \mathrm{O}
$$

$\left[\mathrm{H}_{2} \mathrm{~L}=\mathrm{R}^{\prime} \mathrm{R}^{2} \mathrm{C}=\mathrm{N}(\mathrm{R}) \mathrm{COOH}, \mathrm{R}^{\prime}=\mathrm{H}\right.$ and $\mathrm{CH}_{3}, \mathrm{R}^{2}=2-\mathrm{HOC}_{6} \mathrm{H}_{4} ; \mathrm{R}^{\prime}=\mathrm{CH}_{3}, \mathrm{R}^{2}=\mathrm{HOOC} ; \mathrm{R}$ $=-\mathrm{CH}_{2}-,-\mathrm{CH}_{2}-\mathrm{CH}_{2}->\mathrm{CH}-\mathrm{CH}_{2}-\mathrm{CH}_{3},-\mathrm{CH}_{2}-\mathrm{CH}_{2}-\mathrm{CH}_{2}-,>\mathrm{CH}-\mathrm{CH}\left(\mathrm{CH}_{3}\right)_{2},>\mathrm{CH}-$ $\left.\mathrm{CH}_{2}-\mathrm{CH}_{2} \mathrm{SCH}_{3},>\mathrm{CHC}_{6} \mathrm{H}_{5}(80)\right]$. 


\subsection{PROPERTIES}

\subsubsection{Hydrolytic Stability}

Organotin(IV) adducts and chelates are highly susceptible to hydrolysis (38, 74). However, the chelates are more readily hydrolysed than the adducts and at least in one case the products of hydrolysis have been isolated and identified. Tanaka et al (48) have observed that when nheptane is added to a solution of $\mathrm{Me}_{2}$ Snsalen in reagent grade benzene and the mixture cooled, crystals of $\left(\mathrm{Me}_{2} \mathrm{Sn}\right)$, Osalen are deposited and $\mathrm{H}_{2}$ salen is recovered from the filtrate. When the solution of $\left(\mathrm{Me}_{2} \mathrm{Sn}\right) \mathrm{OSalen}$ is exposed to air for longer periods, it finally changes into $\mathrm{Me}_{2} \mathrm{SnO}$ :
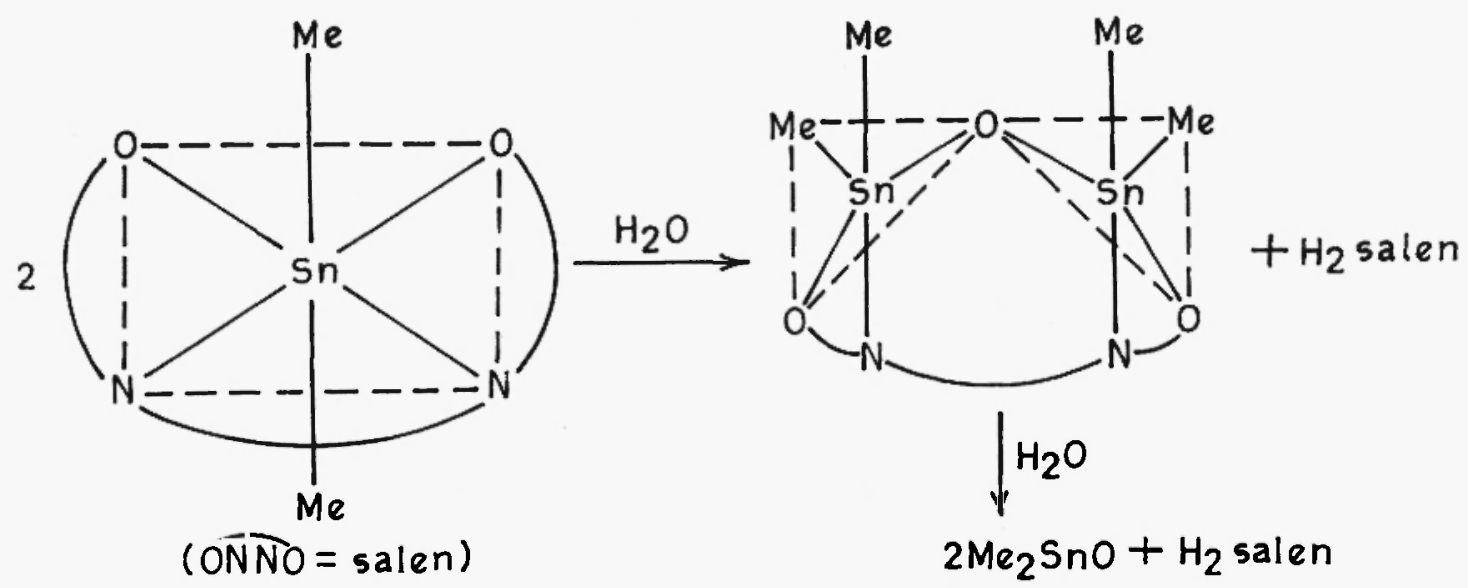

\subsubsection{Volatility and Thermal Stability}

Most of the adducts and chelates of organotin(IV) moieties with the Schiff bases are nonvolatile, crystalline solids which possess sharp melting points $(26,28,29,35-37,51,52,76)$. Di- and tributyltin(IV) derivatives of Schiff bases derived from dithiocarbazate amines are highly viscous liquids $(54,74)$. However, the adducts of dimethyltin(IV) and diethyltin(IV) chlorides with $\mathrm{N}$-alkyl(methyl, ethyl or n-butyl)salicylaldimines (8) are volatile and sublime unchanged in quantitative yields under reduced pressure. Trialkyltin(IV) derivatives of $\mathrm{N}$ : alkyl(aryl)salicylaldimines (60) and 2,2-disubstituted benzothiazolines (70) are also volatile yellow coloured liquids.

\subsubsection{Molecular Association}

Direct molecular weight measurements have been reported for a few adducts and chelates of organotin(IV) halides with the Schiff bases $(35,37,38,53,57,59,74,77)$. Osmometric measurements in methanol solution carried out by Barbieri et al $(19,81)$ on the adducts, $\mathrm{R}_{\mathrm{n}} \mathrm{SnCl}_{4-\mathrm{n}}$. $\mathrm{H}_{2}$ acen, indicate that these dissociate and independent organotin halide, $\mathrm{R}_{\mathrm{n}} \mathrm{SnCl}_{4 \mathrm{n}}$ and ligand, $\mathrm{H}_{2}$ acen, moieties are perhaps present in solution. Trialkyltin(IV) derivatives of $\mathrm{N}$ alkyl(aryl)salicylaldimine (60) and 2,2-disubstituted benzothiazolines (70) as well as the chelates, $\mathrm{R}_{2} \mathrm{SnL}(17,40,48,76,79)$ (where $\mathrm{L}$ is the dianion of a bifunctional terdentate or tetradentate ligand) and $R_{3} S n L$ (where $L$ is the anion of Schiff bases derived from the condensation of dithiocarbazate amines with a ketone or pyridine-2-carboxaldehyde) (54) are monomeric in solution. 
Similarly, the adducts and chelates of tri- and diorganotin(IV) moieties with the Schiff bases derived from the condensation of 2-amino-4-phenylthiazole with different aldehydes have also been reported to be monomeric in nitrobenzene (35). Di- and tri-n-butyltin(IV) chelates of biionic and monoionic Schiff bases are monomeric (73). However, the adducts of organotin(IV) halides with potentially tetradentate neutral ligands have been assigned a polymeric structure in the solid state on the basis of infrared and Mössbauer spectral data (20, 22).

\subsection{STRUCTURAL STUDIES}

Structural aspects of the adducts as well as the chelates of organotin(IV) moieties with the Schiff bases have received much attention. Electronic and nuclear $\left({ }^{1} \mathrm{H},{ }^{13} \mathrm{C}\right.$ and $\left.{ }^{119} \mathrm{Sn}\right)$ magnetic resonance spectral studies along with molecular weight and conductance measurements have been carried out to throw light on the structures of the complexes in solution. On the other hand, infrared, Mössbauer and X-ray crystallographic studies of a few representative complexes have, of course, been made to elucidate the structures of these compounds in the solid state. Conclusions drawn on the basis of these studies are being summarised below:

\subsubsection{Molar Conductance}

Molar conductances of adducts of organotin(IV) chlorides with unidentate, monofunctional bidentate, bifunctional terdentate and bifunctional tetradentate ligands have been measured in nitrobenzene, N,N-dimethylformamide or methanol depending upon the solubility of the adducts and it has been found that the adducts behave as non-electrolytes $(8-10,13,18,25,26,28,29$. $35,36)$. Molar conductance values of the organotin(IV) chlorides and their adducts with $\mathrm{H}_{2}$ acen (81) in methanol at about the same molar concentration have been found to be of the same order. It has been suggested that essentially the same conducting species are present in both systems. In comparison to the adducts, the chelates of organotin(IV) moieties of the Schiff bases show very low values of molar conductance indicating their non-electrolytic nature $(15,17,35,36$, $38,51,52,54,59,78,80)$.

\subsubsection{Electronic Spectra}

Electronic spectra of the adducts $\mathrm{R}_{11} \mathrm{SnCl}_{4-n} \cdot \mathrm{L} \quad(\mathrm{L}=\mathrm{Schiff}$ base ligands $)$ usually correspond with those of the free ligands which strongly suggests that the ligands maintain their keto-amine hydrogen bonded configuration in the complexes also $(28,29,35,36,81)$. On the other hand, the spectra of the chelates of organotin(IV) moieties with different Schiff bases were found to be quite different from those of the free Schiff bases suggesting the deprotonation of the Schiff bases in chelates $(35,36,38,43,51,52,55-57,74,76,80)$. It has been reported that a metal/ metalloid is capable of forming $\mathrm{d} \pi$-p $\pi$ bonds with ligands containing nitrogen as the donor atom. The tin atom has its $5 \mathrm{~d}$ orbitals completely vacant and hence $\mathrm{L} \rightarrow \mathrm{M}$ bonding can take place by the acceptance of a pair of electrons from the nitrogen atom of the ligand. In the electronic spectra of the adducts as well as of chelates. new additional charge transfer bands in the region 240$340 \mathrm{~nm}$ have also been reported $(15,35,36,51,74)$. 


\subsubsection{Infrared Spectra}

Infrared spectra of the adducts as well as of the chelates of organotin(IV) moieties provide valuable informations regarding the structures of the compounds in the solid state. Either an increase or decrease by a few wave numbers in the frequency of $\mathrm{C}=\mathrm{N}$ stretching vibration which appears in the region $1585-1650 \mathrm{~cm}^{-1}$ in the spectra of the free Schiff bases has been generally observed and it has been inferred that the ligands coordinate to the tin atom through the azomethine nitrogen atom $(5,17,24,28,29,35-37,51,52,54,56,73,74)$. Phenolic $v \mathrm{C}-\mathrm{O}$ appearing at $\sim 1280 \mathrm{~cm}^{-1}$ in the IR spectra of the Schiff bases containing $o$-hydroxy group remains almost unaffected in the spectra of organotin(IV) adducts, indicating its non-participation in coordination $(12,17)$. However, in the spectra of organotin(IV) chelates phenolic $v(C-O)$ band is shifted to higher wave number indicating its participation in coordination $(35,36,51,55,59,60,73$, 80). In the spectra of the adducts of organotin(IV) halides with Cosalen, Nisalen and Cusalen, the frequency of phenolic $v \mathrm{C}-\mathrm{O}$ increases from $1540 \mathrm{~cm}^{-1}$ in Msalen to $1550-1560 \mathrm{~cm}^{-1}$ in adducts suggesting coordination of metal chelate to organotin(IV) halide through phenolic oxygen atoms which attain 3-coordination (30-33).

The frequencies of the $\mathrm{Sn}-\mathrm{C}$ stretching vibrations are not affected to any appreciable extent by a change in coordination number of tin, but those of the $\mathrm{Sn}-\mathrm{X}$ stretching modes are very much diminished by an increase in coordination number of tin from 4 to 6 . However, the number of the $v \mathrm{Sn}-\mathrm{C}$ and the $v \mathrm{Sn}-\mathrm{Cl}$ bands appearing in the spectra of the adducts gives an indication of the geometry of the $\mathrm{R}_{\mathrm{n}} \mathrm{SnX}_{4-\mathrm{n}}$ moiety. In the octahedral adducts of $\mathrm{R}_{2} \mathrm{SnCl}_{2}$ with $\mathrm{N}$ alkyl(aryl)salicylaldimines (8, 12), N-alkyl(aryl)-2-hydroxyacetophenoneimines (13), $\mathrm{N}$-alkyl(aryl)2-hydroxy-1-naphthaldimines (14) and N,N'-ethylenebis(acetylacetoneimine) (20); and adducts of $\mathrm{R}_{2} \mathrm{SnX} \mathrm{X}_{2}$ with $\mathrm{N}, \mathrm{N}^{\prime}$-bis(3-nitrobenzylidene)ethylenediamine $\left(\mathrm{X}=\mathrm{Cl}, \mathrm{ClO}_{4}, \mathrm{NO}_{3}, \mathrm{BF}_{4}\right)$ (24), only single bands pertaining to $v_{\text {as }} \mathrm{Sn}-\mathrm{C}$ and $v_{\text {as }} \mathrm{Sn}-\mathrm{Cl}$ or $v_{\text {is }} \mathrm{Sn}-\mathrm{X}$ have been observed which indicate a trans $-\mathrm{R}$, and trans $-\mathrm{Cl}_{2}$ or trans $-\mathrm{X}_{2}$ geometry for the $\mathrm{R}_{2} \mathrm{SnCl}_{2}$ or $\mathrm{R}_{2} \mathrm{SnX}$ moiety. However, in the spectra of 1:1 adducts of $\mathrm{Me}_{2} \mathrm{SnCl}_{2}$ with Nisalen (31) and $\mathrm{Ph}_{2} \mathrm{SnCl}_{2}$ with Schiff bases of S-alkyldithiocarbazate amines (15), only a single band for $v_{\text {as }} \mathrm{Sn}-\mathrm{C}$ at $575 \mathrm{~cm}^{-1}$ and $270 \mathrm{~cm}^{-1}$, respectively, and two bands for $v_{\mathrm{as}} \mathrm{Sn}-\mathrm{Cl}$ and $v_{\mathrm{s}} \mathrm{Sn}-\mathrm{Cl}$ in the range 292-270 and 256-230 $\mathrm{cm}^{-1}$, respectively, have been observed which indicate a trans $-\mathrm{R}_{2}$ and $c i s-\mathrm{Cl}_{2}$ geometry for the adducts.

The infrared spectra of organotin(IV) chelates of the types $\mathrm{R}_{2} \mathrm{SnL}_{\mathrm{N}} / \mathrm{R}_{2} \mathrm{SnL}_{2}$ (where $\mathrm{L}=$ anions of bidentate or tetradentate Schiff bases such as salen, $\mathrm{R}=$ alkyl or aryl) exhibit only one band in the range $570-550 \mathrm{~cm}^{-1}$ for alkyl and $270-229 \mathrm{~cm}^{-1}$ for phenyl which could be assigned to $v_{a s} S n-C$ in a trans-octahedral $R_{2} \operatorname{Sn}(I V)$ moiety $(15,35,51,82,83)$. On the other hand, the spectra of some octahedral $\mathrm{Bu}_{2} \mathrm{SnL}_{2}$ chelates $(\mathrm{L}=$ Schiff base anion) show two bands at 600 and $510 \mathrm{~cm}^{-1}$ for the $\nu_{\mathrm{as}} \mathrm{Sn}-\mathrm{C}$ and $v_{i} \mathrm{Sn}-\mathrm{C}$, respectively, suggesting a cis-Bu, $\mathrm{Sn}(\mathrm{IV})$ moiety in octahedral geometry $(52,73)$.

Two bands due to the $v_{\text {as }} \mathrm{Sn}-\mathrm{C}$ and $v_{s} \mathrm{Sn}-\mathrm{C}$ have been reported for penta-coordinate triorganotin(IV) chelates, $\mathrm{R}_{3} \mathrm{SnL}(\mathrm{L}=$ bidentate Schiff base anion) suggesting that all the three organic groups are not equivalent and do not occupy the equatorial positions $(51,56)$. The spectra 
of some penta-coordinate diorganotin(IV) chelates, $\mathrm{R}_{2} \mathrm{SnL}(\mathrm{L}=$ tridentate Schiff base anion) exhibit two bands due to the $v_{a s} \mathrm{Sn}-\mathrm{C}$ and $\mathrm{v}_{\mathrm{s}} \mathrm{Sn}-\mathrm{C}$ suggesting a bent $\mathrm{R}_{2} \mathrm{Sn}$ moiety (cis-organic groups) in trigonal bipyramidal geometry $(41,43,55,56)$.

\subsubsection{Nuclear $\left({ }^{1} \mathrm{H},{ }^{13} \mathrm{C}\right.$ and $\left.{ }^{119} \mathrm{Sn}\right)$ magnetic resonance spectral studies}

Proton magnetic resonance (PMR) spectra of the adducts of mono-, di- and triorganotin(IV) halides with $\mathrm{H}_{2}$ acen have been studied by Barbieri et al (82) and by Smith and Dodd (84). It has been observed that in $\mathrm{CD}_{3} \mathrm{OD}, \mathrm{CDCl}_{3}$ and $\mathrm{CD}_{2} \mathrm{Cl}_{2}$ solution, the ligand resonances from the adduct are in each case the same as those of the free ligand (84). Similarly, complete dissociation is indicated for $\mathrm{Me}_{3} \mathrm{SnCl} \cdot \mathrm{H}_{2}$ acen and $\mathrm{Me}_{2} \mathrm{SnCl}_{2} \cdot \mathrm{H}_{2}$ acen by the coincidence of the tin-methyl proton resonance signals and ${ }^{117} \mathrm{Sn}-\mathrm{CH}_{3}$ and ${ }^{119} \mathrm{Sn}-\mathrm{CH}_{3}$ coupling constants measured for the organotin(IV) chlorides and their $\mathrm{H}_{2}$ acen adducts (81). Their osmometric measurements have also indicated the complete dissociation $(19,81)$

Recently, Khoo et al have reported (26) that in the PMR spectra of $\mathrm{Ph}_{3} \mathrm{SnX}$.L (where X $=\mathrm{Cl}$ or $\mathrm{NCS}, \mathrm{L}=\mathrm{N}$-arylsalicylideneimines and $\mathrm{N}$-aryl-2-hydroxynaphthylideneimines) the sharp singlet due to the azomethine proton of the ligands $(\delta 8.50-9.20 \mathrm{ppm})$ remains unchanged on coordination, indicating that the $\mathrm{N}$-atom is not coordinated to the metal. On the other hand, the peak due to the phenolic proton of the ligands ( $\delta 13.00-15.00 \mathrm{ppm})$ becomes significantly broadened on complex formation. Coordination through oxygen is expected to result in a weakening of the $\mathrm{O}-\mathrm{H}$ bond, and a strengthening of the $\mathrm{C}=\mathrm{N}---\mathrm{H}$ hydrogen bond, which in turn leads to the observed broadening of the peak due to phenolic proton. Similar observations have also been reported for the complexes of dimethyltin dichloride with $\mathrm{N}$-arylsalicylideneimines (27). The coordination through oxygen has also been confirmed by the comparison of the ${ }^{13} \mathrm{C} N \mathrm{NR}$ spectral data of the ligands such as $\mathrm{N}$-salicylideneaniline and $\mathrm{N}$-salicylidene- $p$-methoxyaniline, and their $\mathrm{Me}_{2} \mathrm{SnCl}_{2}$ complexes (27). The azomethine carbon signal remains unchanged whereas the signal due to the carbon attached to the phenolic oxygen moves downfield ( $\delta$ 0.4-0.6 ppm) indicating coordination through oxygen. The coupling constants ${ }^{2} \mathrm{~J}\left({ }^{119} \mathrm{Sn}-\mathrm{CH}_{3}\right)$ determined for dimethyltin dichloride $\mathrm{N}$-salicylideneaniline and dimethyltin dichloride $\mathrm{N}$-salicylidene- $p$-methoxyaniline are 70.8 and $71.2 \mathrm{~Hz}$, respectively (27).

PMR spectra of dimethyltin(IV) chelates of bifunctional terdentate ligands having ONO and SNO donor atom systems have been studied by Barbieri et al (43) and on the basis of position of $\mathrm{Me}_{2} \mathrm{Sn}(\mathrm{IV})$ signals and the value of $\mathrm{J}\left({ }^{117} \mathrm{Sn}-\mathrm{CH}_{3}\right.$ and $\left.{ }^{119} \mathrm{Sn}-\mathrm{CH}_{3}\right)$, a trigonal bipyramidal structure with a bent $\mathrm{Me}_{2} \mathrm{Sn}(\mathrm{IV})$ moiety has been proposed for these complexes. A series of dimethyltin(IV) chelates of bifunctional terdentate ligands prepared by the condensation of substituted salicylaldehydes with substituted 2-aminophenol has been studied by PMR spectroscopy in a number of weak and strong donor solvents by Tanaka et al (45). The coupling constants between tin-119 and the methyl protons attached directly to the tin atom $\left(\mathrm{J}{ }^{119} \mathrm{Sn}-\mathrm{CH}_{3}\right)$, and the average value of those between tin-117 and tin-119 nuclei and the azomethine proton ( $\mathrm{J}$ ${ }^{117 / 119} \mathrm{Sn} \leftarrow \mathrm{N}=\mathrm{C}-\mathrm{H}$ ) through three bonds including the $\mathrm{N} \rightarrow \mathrm{Sn}$ bond have been measured in $\mathrm{CDCl}_{3}$ 
and DMSO- $\mathrm{d}_{6}$. Distinct values of $\mathrm{J}\left({ }^{117 / 119} \mathrm{Sn} \leftarrow \mathrm{N}=\dot{\mathrm{C}}-\mathrm{H}\right)$ of the order of $45.9-50.0 \mathrm{~Hz}$ and 30.5 $47.9 \mathrm{~Hz}$ in $\mathrm{CDCl}_{3}$ and DMSO- $\mathrm{d}_{6}$, respectively suggest that the complexes do not dissociate and maintain five- coordination around tin(IV) even in strong donor solvents. Low $\mathrm{J}\left({ }^{119} \mathrm{Sn}-\mathrm{CH}_{3}\right)$ values $(78.0-85.0 \mathrm{~Hz})$ in weak donor solvents like $\mathrm{CDCl}_{3}, \mathrm{CH}_{3} \mathrm{NO}_{2}$ and $\left(\mathrm{CH}_{3}\right)_{2} \mathrm{CO}$ correspond to a bent $\mathrm{Me}_{2} \mathrm{Sn}(\mathrm{IV})$ moiety in a trigonal bipyramidal arrangement whereas $\mathrm{J}\left({ }^{119} \mathrm{Sn}-\mathrm{CH}_{3}\right)$ values as high as 93.0-109.8 Hz obtained in strong donor solvents (DMSO- $d_{6}$ and HMPA) correspond to almost a linear $\mathrm{Me}_{2} \mathrm{Sn}(\mathrm{IV})$ species in an octahedral arrangement. It has been reported that an initially bent $\mathrm{C}-\mathrm{Sn}-\mathrm{C}$ moiety in a 5-coordinate environment present in poor donor solvents changes to an almost linear $\mathrm{C}-\mathrm{Sn}-\mathrm{C}$ geometry due to the coordination of a molecule of the strong donor solvent, thereby, raising the coordination number of tin from 5 to 6 (Eq.50).
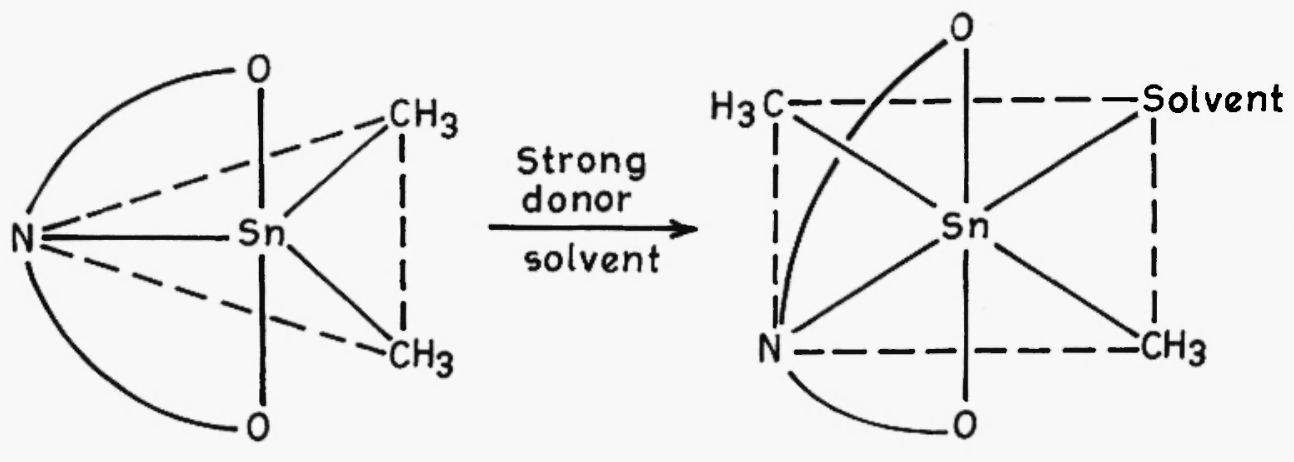

The PMR spectrum of N,N'-ethylenebis(salicylaldiminato)dimethyltin(IV), $\mathrm{Me}_{2}$ Snsalen, was studied by Murray et al (21) and Tanaka et al (23) in $\mathrm{CDCl}_{3}$ solution. Murray et al (21) observed two distinct sharp lines at $\delta 0.68,0.92 ; 3.70,3.89$ and $7.98,8.32 \mathrm{ppm}$ for each group of $\mathrm{Me}_{2} \mathrm{Sn}(\mathrm{IV}),-\mathrm{CH}_{2} \mathrm{CH}_{2}-$ and $-\mathrm{N}=\mathrm{CH}-$ protons. However, Tanaka et al (23) reported two lines only for the $\mathrm{Me}_{2} \mathrm{Sn}(\mathrm{IV})$ protons at $\delta 0.72$ and $0.75 \mathrm{ppm}$ whereas the $-\mathrm{CH}_{2} \mathrm{CH}_{2}-$ and $-\mathrm{CH}=\mathrm{N}-$ protons appeared as singlets at $\delta 4.07$ and $8.07 \mathrm{ppm}$, respectively. Although Tanaka and coworkers suggested the existence of two isomers (cis/trans) for the complex at least in solution, Murray and co-workers proposed a cis-structure having a non-planar salen ${ }^{-2}$ moiety. Later on, Tanaka et al (48) succeeded in isolating the two isomers in the solid state and studied the cis/ trans isomerization in a number of solvents. The value of equilibrium constant $\mathrm{K}$ for the cis/

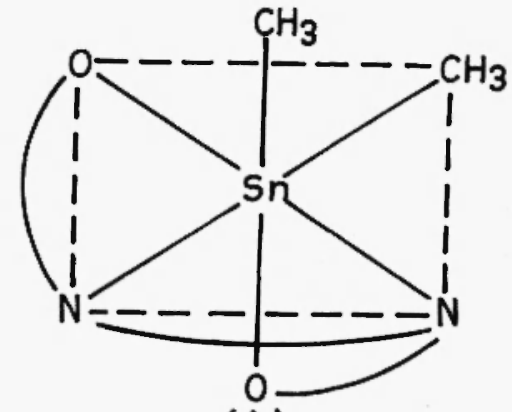

(A)

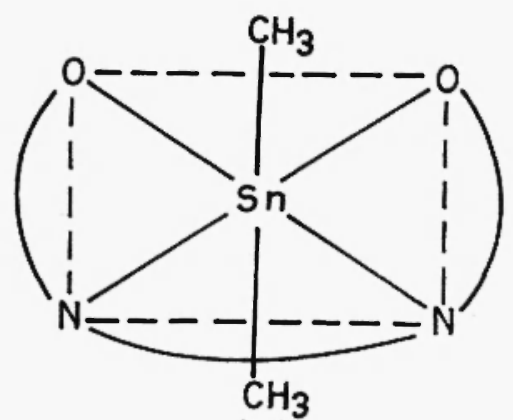

(B)

Fig. 1: Structures of cis- (A) and trans- (B) isomers of Me $\mathrm{e}_{\mathbf{i}}$ Snsalen. 
trans isomerisation reaction and the variable temperature PMR spectra indicated the cis-isomer [Fig. $1(\mathrm{~A})$ ] to be favoured in non-coordinating solvents $\left(\mathrm{CH}_{2} \mathrm{Cl}_{2}\right.$ and $\left.\mathrm{C}_{6} \mathrm{H}_{5} \mathrm{NO}_{2}\right)$ and at room temperature whereas the trans-isomer (Fig 1(B)] predominated in strong donor solvents (DMA and HMPA) and at higher temperatures.

In the PMR spectra of organotin(IV) chelates of various Schiff bases $(28,35-38,51-53$, $55,56,71,73,74,77-79$ ) slight downward chemical shift in the value of $\delta-\mathrm{CH}=\mathrm{N}-$ protons may arise due to the participation of the azomethine nitrogen in chelation. Disappearance of $\mathrm{OH},-\mathrm{NH}$ or $-\mathrm{SH}$ signals of mono- or bifunctional Schiff bases in the PMR spectra of their organotin(IV) chelates indicates the chelation of the ligand moiety through deprotonated oxygen, nitrogen or sulphur atom $(15,35,36,38,51-53,56-58,73,74,78)$. On the basis of the PMR spectral data, the complexes of trialkyltin(IV) species with monofunctional bidentate Schiff bases such as $\mathrm{N}$-alkyl(aryl)salicylaldimines (60) and 2,2-disubstituted benzothiazolines (70) have been assigned five-coordinated structures with trigonal bipyramidal geometries (Fig. 2).

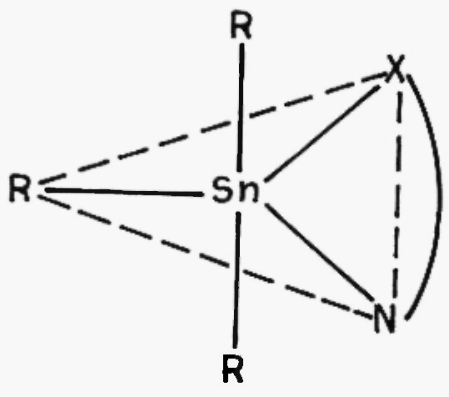

$X=0$ or $S$

mer

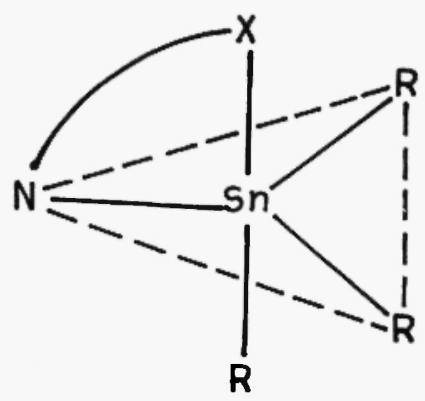

$X=0$ or $S$

all cis

Fig. 2: Structures of $R_{3} \operatorname{Sn}(X N)$, [where $X N=N$-alkyl(aryl)salicylaldimines or 2,2disubstituted benzothiazolines].

${ }^{13} \mathrm{C}$ and ${ }^{119} \mathrm{Sn}$ NMR spectra of dibutyltin(IV), tributyltin(IV) or trimethyltin(IV) chelates of monofunctional bidentate and bifunctional terdentate Schiff bases having NO or NS and ONO or SNO donor systems, respectively, have been studied by Tandon et al $(53,73,74,79)$. The ${ }^{13} \mathrm{C}$ chemical shifts for carbons attached to $\mathrm{O}, \mathrm{S}$ and $\mathrm{N}$ indicate the involvement of $\mathrm{O}, \mathrm{S}$ and $\mathrm{N}$ in coordination. The values of ${ }^{1}\left({ }^{119} \mathrm{Sn}-{ }^{13} \mathrm{C}\right)$ in these complexes in the range $607.7-555.0 \mathrm{~Hz}$ and ' $\mathrm{J}\left({ }^{117} \mathrm{Sn}-{ }^{13} \mathrm{C}\right)$ at $570.2 \mathrm{~Hz}$ only for $\mathrm{Bu}_{2} \mathrm{SnL}(\mathrm{L}=$ anion of semicarbazone of benzaldehyde) indicate the five-coordination around tin. Further, these complexes give sharp signals between -100 and $190 \mathrm{ppm}$ in ${ }^{119} \mathrm{Sn} \mathrm{NMR}$, which also support the five-coordination around tin in a trigonal bipyramidal geometry. The value of ${ }^{\prime} \mathrm{J}\left({ }^{119} \mathrm{Sn}-{ }^{13} \mathrm{C}\right)$ has also been used to calculate $\mathrm{C}-\mathrm{Sn}-\mathrm{C}$ angles which are $140-143^{\circ}$ in the octahedral complexes, $\mathrm{R}_{2} \mathrm{SnL}$ [where $\mathrm{L}=$ dianion of S-methyl- $\beta-\mathrm{N}$ (substituted phenyl)methylendithiocarbazate; $\mathrm{R}=\mathrm{CH}_{2} \mathrm{CH}_{2} \mathrm{CO}_{2} \mathrm{Me}, \mathrm{CH}_{2} \mathrm{CH}_{2} \mathrm{CO}_{2} \mathrm{Et}, \mathrm{CH}_{2} \mathrm{CH}_{2} \mathrm{CO}_{2} \mathrm{Bu}$ ] (57). 


\subsection{5. ${ }^{119} \mathrm{Sn}$ Mössbauer and X-ray crystallographic studies}

${ }^{119} \mathrm{Sn}$ Mössbauer spectroscopy has been frequently employed to throw light on the structures of Schiff base and $\beta$-ketoamine complexes of organotin(IV) species and the data have been reviewed in two articles $(85,86)$.

Barbieri et al $(20,22)$ have studied Mössbauer spectra of the adducts of organotin(IV) halides with potentially tetradentate $\mathrm{N}, \mathrm{N}$ '-ethylenebis(acetylacetoneimine) and $\mathrm{N}, \mathrm{N}^{\prime}$-ethylenebis(salicylaldimine). Large values of quadrupole splitting (Q.S.) $\left(3.62-4.49 \mathrm{~mm} \mathrm{~s} \mathrm{~s}^{-1}\right.$ ) and isomeric shift (I.S.) (1.26-1.62 $\mathrm{mm} \mathrm{s}^{-1}$ ) along with the temperature dependence of the Mössbauer parameters indicate a polymeric all trans-octahedral structure for the adducts of diorganotin(IV) halides in the solid state in which the planar $\mathrm{R}_{2} \mathrm{SnX}_{2}$ moieties are bridged by the trans-isomer of the bismonodentate ligand. The all trans-octahedral polymeric structure of $\mathrm{Me}_{2} \mathrm{SnCl}_{2} \cdot \mathrm{H}_{2}$ salen has been further confirmed by Randaccio (87) with the help of X-ray crystallography.

Point charge model calculations have also been done to deduce the configuration of the adducts, $\mathrm{RSnCl}_{3} \cdot \mathrm{H}_{2}$ salen ( $\mathrm{R}=\mathrm{Me}, \mathrm{Oct}^{\prime \prime}$ and $\mathrm{Ph}$ ) (22) and $\mathrm{Me}_{3} \mathrm{SnCl} . \mathrm{H}_{2}$ acen (88). It has been reported that these adducts are octahedral and possess polymeric structure in which $\mathrm{Me}_{3} \mathrm{SnCl}$ and $\mathrm{RSnCl}_{3}$ moieties with $\mathrm{T}$ shaped $\mathrm{Me}_{3} \mathrm{Sn}$ and $\mathrm{SnCl}_{3}$ geometry are bridged by two separate ligand moieties.

Mössbauer spectra of the adducts of $\mathrm{R}_{2} \mathrm{SnCl}_{2}$ and $\mathrm{RSnCl}_{3}$ with $\mathrm{Msalen}(\mathrm{M}=\mathrm{Ni}$ and $\mathrm{Cu})$ have also been studied (30-33). It has been suggested that the chelates, Msalen, act as a bidentate oxygen donor ligand and the unimolecular adducts possess an octahedral structure having trans$\mathrm{R}_{2}$, cis- $\mathrm{Cl}_{2}$ geometry in $\mathrm{R}_{2} \mathrm{SnCl}$, adducts and $\mathrm{T}$ shaped $\mathrm{SnCl}_{3}$ moiety in the adducts of $\mathrm{RSnCl}_{3}$. The structure of $\mathrm{Me}_{2} \mathrm{SnCl}_{2}$.Nisalen (Fig. 3) has been further confirmed by X-ray crystallography (89).

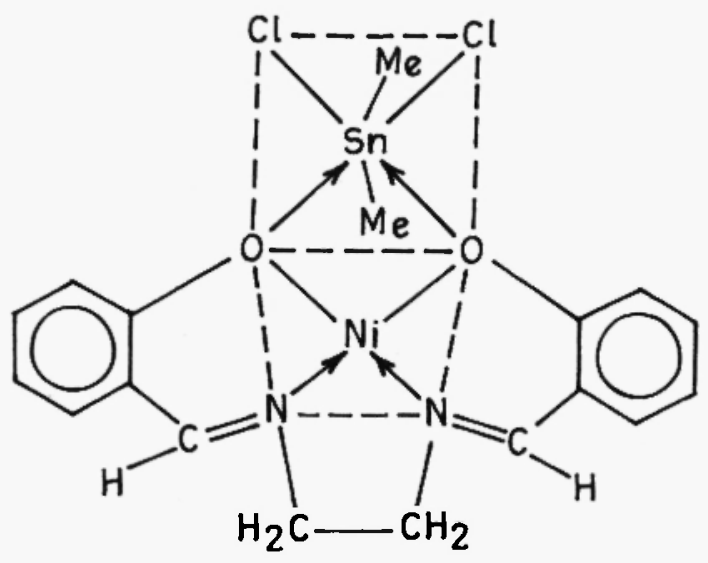

Fig. 3: Structure of $\mathrm{Me}_{2} \mathrm{SnCl}_{2}$. Nisalen.

It has been reported (26) that the adducts of triphenyltin(IV) chloride or isothiocyanate with the Schiff bases such as $\mathrm{N}$-arylsalicylideneimines and $\mathrm{N}$-aryl-2-hydroxynaphthylideneimines have the Q.S. 3.14-3.38 $\mathrm{mm} \mathrm{s} \mathrm{s}^{-1}$ which support the trigonal bipyramidal geometry with three phenyl groups in the equatorial plane. This conclusion has been further confirmed (26) by the X-ray 
structural determination of isothiocyanatotriphenyl(I-[(4'-methylphenylimino)-methyl]-2naphthol)tin(IV). This complex has a trigonal bipyramidal structure, with the three phenyl groups occupying the equatorial positions around the tin atom, while the $\mathrm{O}$ atom of the ligand and the $\mathrm{N}$ of the NCS group occupy the axial positions (Fig. 4). The Sn atom is slightly distorted from equatorial plane defined by the three bonding $C$ atoms of the phenyl groups $\left(0.050(4) \mathrm{A}^{\circ}\right)$. The average $\mathrm{Sn}-\mathrm{C}(\mathrm{Ph})$ distance is $2.115(6) \mathrm{A}^{\circ}$ and the $\mathrm{O}-\mathrm{Sn}-\mathrm{N}$ angle is $177.94(14)^{\circ}$. The NCS group is almost linear, the NCS angle being $178.1(5)^{\circ}$ and the $\mathrm{Sn}-\mathrm{N}, \mathrm{N}-\mathrm{C}$ and $\mathrm{C}-\mathrm{S}$ distances are $2.230(5), 1.144(6)$ and $1.605(6) \mathrm{A}^{\circ}$, respectively. A striking feature of the structure is that the phenolic proton of the ligand has moved to the imine nitrogen and the Schiff base is thus
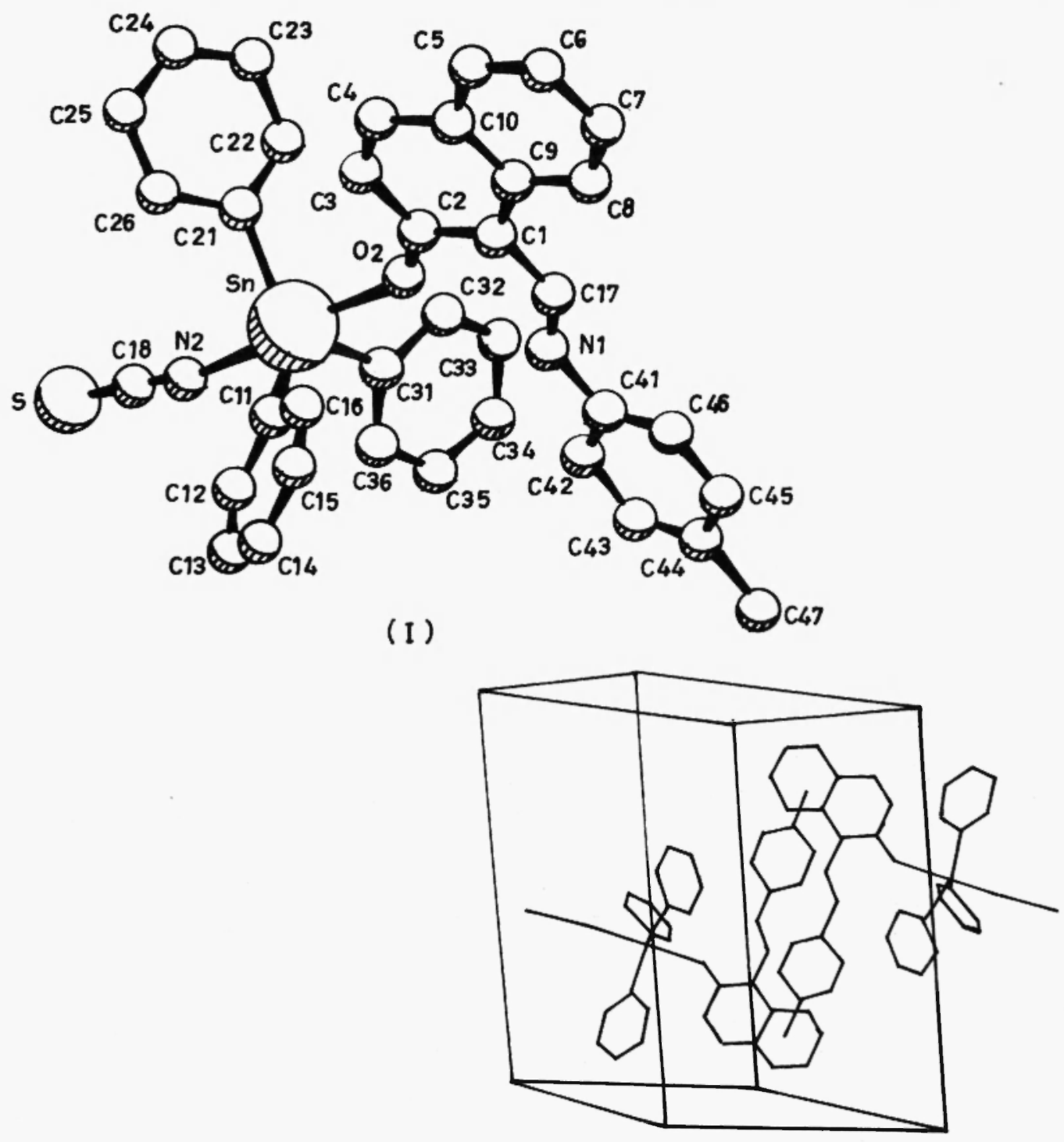

(II)

Fig. 4: Molecular configuration (I) and stereoview of packing arrangement(II) of isothiocyanatotriphenyl- (1-[(4'-methylphenylimino)-methyl]-2-naphthol)tin(IV). 
coordinated in the form of a zwitter ion. It has been reported (26) that the complexes pack together in the crystal in pairs of centrosymmetrically - related dimers, with the Schiff bases lying flat, one above the other, and head-to-tail relative to each other, as shown in Fig. 4(II). The coordination of the Schiff bases through oxygen, not through the azomethine nitrogen has also been confirmed by the X-ray structural analysis of the complex of dimethyltin dichloride with 2-( $p$-methoxyphenyliminomethyl)phenol (90).

Barbieri et al have also studied the Mössbauer spectra of chelates $\mathrm{R}_{2} \mathrm{SnL}$ (91) derived from bifunctional terdentate Schiff bases and the complexes depict trigonal bipyramidal geometry. X-ray crystallographic data have further shown that the three $\mathrm{R}_{2} \mathrm{SnL}$ complexes, $\mathrm{Me}_{2} \mathrm{Snsa}$ ap (92, 93), $\mathrm{Ph}_{2}$ Snsalap (94), and $\mathrm{Ph}_{2}$ Snsalatp (95) [where salap and salatp are the dianions of $\mathrm{N}$ 2-hydroxyphenylsalicylaldimine and 2-(2-hydroxyphenyl)benzothiazoline, respectively] possess distorted trigonal bipyramidal structure with the azomethine nitrogen atom and both carbon atoms (attached to tin) occupying equatorial sites; the axial sites being occupied by the other two ligand atoms $(\mathrm{O}, \mathrm{O}$ or $\mathrm{O}, \mathrm{S})$.

The Mössbauer data of triphenyltin(IV) chelates of monofunctional bidentate semicarbazones or thiosemicarbazones suggest trigonal bipyramidal geometry with phenyl groups occupying two equatorial and one axial sites (51).

On the basis of Mössbauer data of the chelates RSnClL (where $\mathrm{L}$ is the dianion of bifunctional terdentate Schiff bases having ONO or ONS donor atom system) (68), it has been suggested that the chelates possibly have polymeric trigonal bipyramidal structure although the monomeric trigonal bipyramidal and oxygen or sulphur bridged dimeric octahedral geometries can not be ruled out completely.

Mössbauer spectra of $\mathrm{Me}_{2}$ Snsalen and other homologous compounds $\mathrm{R}_{2}$ Snsalen have been studied $(47,83)$. Slightly lower Q.S. $\left(3.46 \mathrm{~mm} \mathrm{~s}^{1}\right)$ and I.S. $\left(1.13 \mathrm{~mm} \mathrm{~s}^{1}\right)$ values indicate a distorted trans $-\mathrm{R}_{2}$ structure with $\mathrm{C}-\mathrm{Sn}-\mathrm{C}$ angle less than $180^{\circ} \mathrm{C}$ (Fig. 5). The crystal and molecular structure of the compound, $\mathrm{Me}_{2} \mathrm{Snsalen}$, has also been determined by Randaccio and co-workers (96) and $\mathrm{C}-\mathrm{Sn}-\mathrm{C}$ angle in $\mathrm{Me}_{2} \mathrm{Snsalen}$ has actually been found to be $160^{\circ}$.

The Mössbauer data of diorganotin(IV) chelates, $\mathrm{Bu}_{2} \mathrm{SnL}$ ( $\mathrm{L}=$ dianions of thiosemicarbazones) suggested octahedral geometry with cis- $\mathrm{R}$ groups $(36,52,73,79)$. X-ray structural studies on the compound, $\mathrm{Bu}_{2} \mathrm{Sn}\left[\mathrm{OC}_{6} \mathrm{H}_{4} \mathrm{CH}=\mathrm{NNC}(\mathrm{S}) \mathrm{NH}_{2}\right]$, revealed monoclinic lattice with $\mathrm{a}=16.90 \mathrm{~A}^{\circ}, \mathrm{b}=9.71 \mathrm{~A}^{\circ}, c=8.60 \mathrm{~A}^{\circ}$, and $\beta=103^{\circ} .45^{\prime}$ (73).

Ruddick and Sams $(47,97,98)$ have studied the magnetically perturbed Mössbauer spectra of N-2-hydroxyphenylsalicylaldiminatotriphenyltin(IV), $\mathrm{Ph}_{3} \mathrm{SnHsalap}$. On the basis of positive sign of quadrupole coupling constant and the value of asymmetric parameter of the electric field gradient which is close to unity, an uncommon mer-octahedral structure (Fig. 6) has been assigned to the complex.

Mehrotra et al have also applied the Mössbauer technique to elucidate the structures of the adducts of dimethyltin(IV), dibutyltin(IV), ethyltin(IV) and butyltin(IV) chlorides with N- 

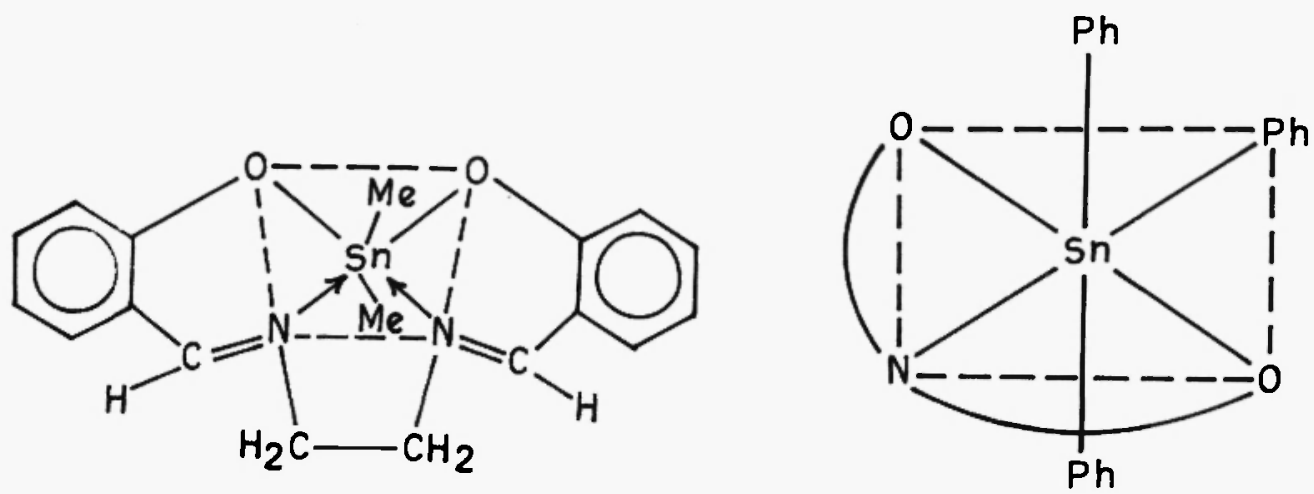

Fig. 5: Structure of $\mathrm{Me}_{2}$ Snsalen.

Fig. 6: Structure of $\mathrm{Ph}_{3} \mathrm{SnHsalap.}$

alkyl(aryl)salicylaldimines(8), N-alkyl(aryl)-2-hydroxyacetophenoneimines (13), Nhydroxyalkylsalicylaldimines (18) and N,N'-alkylenebis(2-hydroxyacetophenoneimines) (25). From the observed large quadrupole splittings $\left(3.95-4.44 \mathrm{~mm} \mathrm{~s}^{-1}\right)$ and isomeric shifts $(1.25-1.61 \mathrm{~mm} \mathrm{~s}$

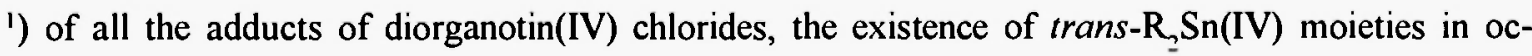
tahedral arrangement around tin atom has been suggested for the above complexes. Comparatively, smaller values of Q.S. (1.76-2.11 $\left.\mathrm{mm} \mathrm{s}^{1}\right)$ and I.S. $\left(0.78-0.89 \mathrm{~mm} \mathrm{~s}^{1}\right)$ in the adducts of $\mathrm{EtSnCl}_{3}$ appear to indicate, on the other hand, a T-shaped $\mathrm{SnCl}_{3}$ moiety in octahedral geometry.

\subsection{MASS SPECTRAL STUDIES}

The mass spectral fragmentation schemes of the $\mathrm{Bu}_{2} \mathrm{Sn}\left[\mathrm{OC}_{6} \mathrm{H}_{4} \mathrm{C}\left(\mathrm{CH}_{3}\right)=\mathrm{NNC}(\mathrm{S}) \mathrm{NH}_{2}\right]$, $\mathrm{Bu}_{2} \mathrm{Sn}\left[\mathrm{OC}_{6} \mathrm{H}_{4} \mathrm{CH}=\mathrm{NNC}(\mathrm{S}) \mathrm{NH}_{2}\right], \mathrm{Bu}_{2} \mathrm{Sn}\left[\mathrm{OC}_{6} \mathrm{H}_{4} \mathrm{C}\left(\mathrm{CH}_{3}\right)=\mathrm{NNC}=\mathrm{S}\left(\mathrm{SCH}_{3}\right)\right], \mathrm{Bu}_{2} \mathrm{Sn}\left[\mathrm{OC}_{6} \mathrm{H}_{4} \mathrm{CH}=\right.$ $\left.\mathrm{NNC}=\mathrm{S}\left(\mathrm{SCH}_{2} \mathrm{C}_{6} \mathrm{H}_{5}\right)\right]$ and $\mathrm{Bu}_{3} \mathrm{Sn}\left(\mathrm{OC}_{6} \mathrm{H}_{4} \mathrm{CH}=\mathrm{NC}_{6} \mathrm{H}_{4} \mathrm{~F}\right)$ have been reported by Tandon et al (72$74,79)$. The fragmentation starts with the simple cleavage of a $\mathrm{Sn}-\mathrm{Bu}$ bond eliminating $\mathrm{Bu}$ of 57 a.m.u. leading to the formation of a three-coordinate $\mathrm{Sn}$ fragment ion. The $\mathrm{Sn}-\mathrm{S}$ bond is more readily cleaved than the $\mathrm{Sn}-\mathrm{O}$ bond showing, thereby, the strong nature of the latter. Some low abundance ions containing $\mathrm{Sn}-\mathrm{S}$ at $\mathrm{m} / \mathrm{e} 15 \mathrm{l}$ are also obtained by the processes which do not involve cleavage of bonds to metal. The last step in the fragmentation is the removal of a HCN molecule, which is characteristic of the Schiff base complexes.

\subsection{THERMAL STUDIES}

A survey of the existing literature revealed that very little work has been done on the thermal decomposition of organotin(IV) compounds. The interpretation of pyrolysis reactions involved in the decomposition of various organotin(IV) derivatives has been shown to be difficult because of the lack of informations on the thermal decomposition of simple organotin(IV) compounds. Thermogravimetric analysis of diorganotin(IV) complexes of Schiff bases obtained by the condensation of aromatic aldehyde with $o$-arsanilic acid and 1,2-ethylenediamine with acetylacetone have been carried out (77). No proper conclusion has been made from the weight loss except the loss of water upto $180^{\circ} \mathrm{C}$. Recently, thermal decomposition scheme of triphenyltin chloride benzilsemicarbazone has been reported from our laboratory in the temperature range 27 $715^{\circ} \mathrm{C}$ using TG, DTG and DTA techniques (99). The decomposition of the complex takes place 
in four steps in the temperature range $127-550^{\circ} \mathrm{C}$, giving $\mathrm{SnO}_{2}$ as end product. The thermal decomposition of $\mathrm{Ph}_{3} \mathrm{SnCl}(\mathrm{Benz} . \mathrm{SCZ})$ is shown in Fig.7

Recently, thermal decomposition of $\mathrm{Ph}_{3} \mathrm{SnCl}(\mathrm{L}-\mathrm{I}), \mathrm{Ph}_{2} \mathrm{SnCl}_{2}(\mathrm{~L}-2)$ and $\mathrm{Me}_{3} \mathrm{Sn}(\mathrm{L}-3)$ (35), where (L-1), (L-2) and (HL-3) are Schiff bases derived from the condensation of 2-amino-4phenylthiazole with 4-methoxybenzaldehyde, furfuraldehyde and salicylaldehyde, respectively, and $\mathrm{Ph}_{2} \mathrm{SnCl}_{2}(\mathrm{~L}-1), \mathrm{Ph}_{3} \mathrm{SnCl}(\mathrm{L}-2), \mathrm{Ph}_{2} \mathrm{Sn}(\mathrm{L}-3)$ and $\mathrm{Ph}_{3} \mathrm{Sn}(\mathrm{L}-4)(29,52)$, where (L-1), (L-2), (L-3) and (HL-4) are the Schiff bases derived from the condensation of thiosemicarbazide with acetophenone, glyoxal, 5-chlorosalicylaldeyde and 4-nitrobenzaldehyde, respectively, and $\mathrm{Ph}_{3} \mathrm{SnCl}(\mathrm{HL}-1), \mathrm{Ph}_{2} \mathrm{Sn}(\mathrm{L}$ 1) ${ }_{2}, \mathrm{Me}_{3} \mathrm{SnCl}(\mathrm{L}-2), \mathrm{Ph}_{3} \mathrm{SnCl}(\mathrm{L}-3)$ and $\mathrm{Ph}_{3} \mathrm{Sn}(\mathrm{L}-4)$ (36), where (HL-1), (L-2), (L-3) and (HL-4) are the Schiff bases derived from the condensation of 2-amino-5-(o-anisyl)-1,3,4-thiadiazole with 2-hydroxynaphthaldehyde, benzylmethyl ketone, acetylacetone and salicylaldehyde, respectively, have been reported from our laboratory and in each case the end product is either $\mathrm{SnO}_{2}$ or $\mathrm{SnS}$ which has been confirmed by IR spectra and X-ray determination.

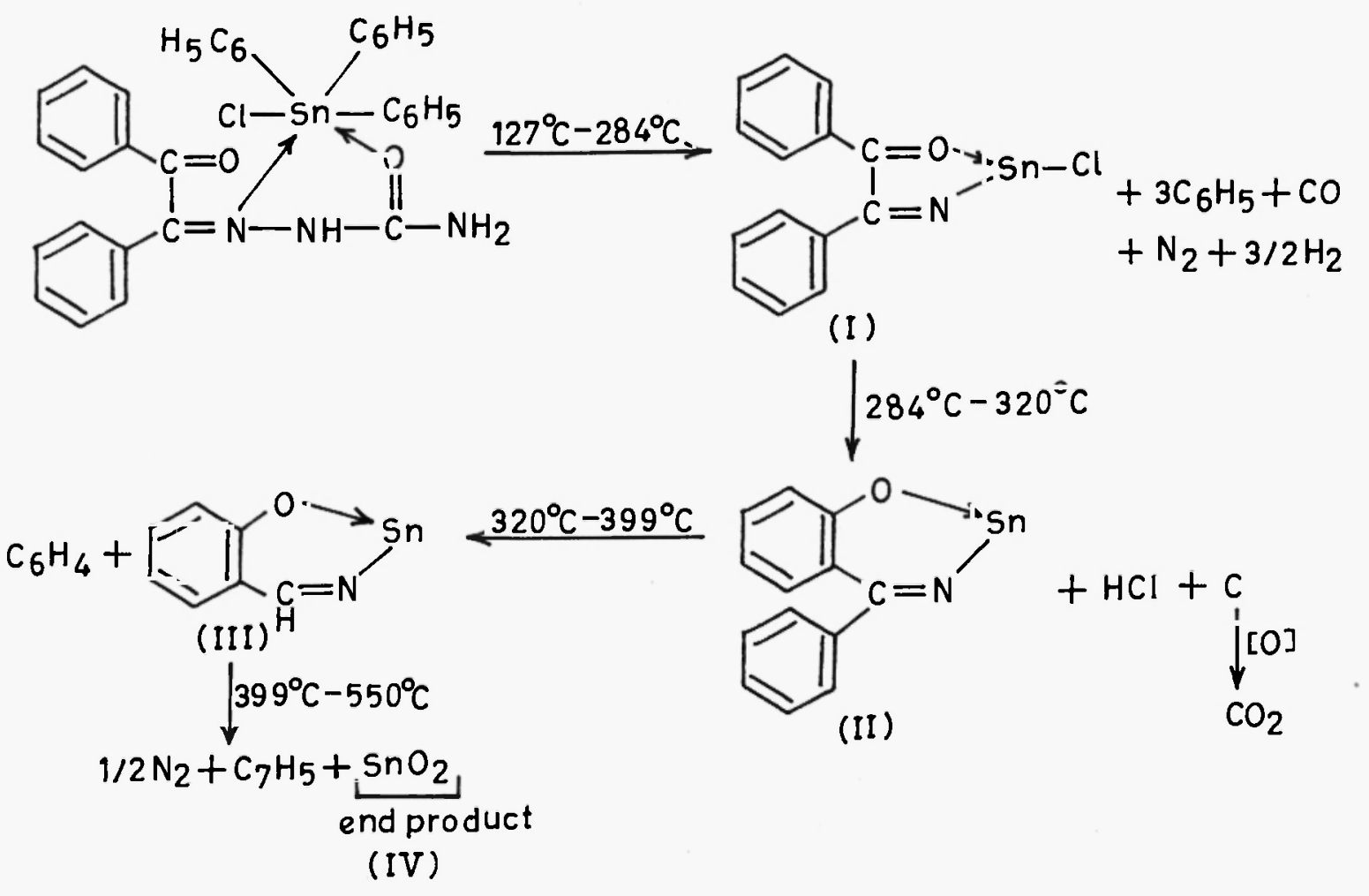

Fig. 7: Proposed scheme for decomposition of $\mathrm{Ph}_{3} \mathrm{SnCl}(\mathrm{Benz} . \mathrm{SCZ})$.

Thermal decompositions of chloro(2-furanyl)semicarbazonotriphenyltin(IV), (2hydroxyphenyl)methylsemicarbazonodiphenyltin(IV), (4-methoxyphenyl)thiosemicarbazonotriphenyltin(IV) (100), S-benzyl- $\beta$-N-(2-hydroxyphenyl)methylendithiocarbazatodiphenyltin(IV), Sbenzyl- $\beta-N-($ benzoylphenyl)methylendithiocarbazatotriphenyltin(IV) and N-methyl-S-benzyl- $\beta$-N-(2hydroxyphenylethylidene)dithiocarbazatotriphenyltin(IV) have also been reported from our laboratory and in each case the end product is either $\mathrm{SnO}_{2}$ or mixture of $\mathrm{SnO}+\mathrm{S}$ or $\mathrm{SnS}$, which has been confirmed by X-ray determination (101). 


\subsection{ANTIMICROBIAL ACTIVITY}

Only scanty references are available on the antimicrobial activity of organotin(IV) Schiff base complexes. Organotin(IV) complexes of Schiff bases containing $S$ and $F$ are potentially active at low doses on axenically grown Entamoeba histolytica trophozoities (strain NIH-20). Tri-nbutyltin(2-fluorobenzaldehyde)-S-benzyldithiocarbazate shows remarkable activity at an extremely low dose even after $48 \mathrm{hr}$ (102).

The antifungal activities of trimethyltin(IV) complexes of heterocyclic thiosemicarbazones have been evaluated against different pathogenic fungi (53). Di- and Tri-n-butyltin(IV) chelates of monofunctional bidentate or bifunctional tridentate Schiff bases derived from salicylaldehyde/ 2-hydroxy-l-naphthaldehyde with fluoroaniline and benzaldehyde/2-hydroxyacetophenone with $o$ aminothiophenol are highly active towards pathogenic bacteria (79). None of the chelates or Schiff bases is active against gram-negative bacterium such as Escherichia coli whereas all the chelates show marked activity against gram-positive bacteria (Bacillus subtilis and Staphylococcus aureus) as compared to the corresponding Schiff bases, which suggest that the complex formation increasing the activity (79). The complexes of the type $\mathrm{n}-\mathrm{Bu}_{2} \mathrm{SnL}$ (where $\mathrm{L}=$ dianion of $\mathrm{S}$-methyl$\beta$-N-(2-hydroxyphenyl)methylendithiocarbazate) are found to be active against P388 lymphocytic leukemia (74).

Antimicrobial activity of di- and triorganotin(IV) complexes of thio Schiff bases derived from the condensation of 2-amino-4-phenylthiazole (35), thiosemicarbazide $(29,52)$ and 2-amino5-(o-anisyl)-1,3,4-thiadiazole (36) with different aldehydes or ketones have been carried out against E. coli, B. subtilis, S. typhi, A. terrus, C. falcatum Went (35) and Streptococcus faecalis, Klebisiella pneumoniae, Escherichia coli, Pseudomonas aeruginosa, Staphylococcus aureus Penicillin resistance (2500 units), Candida albicans, Cryptococcus neoformans, Sporotrichum schenkii, Trichophyton mentagrophytes and Aspergillus fumigatus (29, 36, 52). All these complexes have remarkable antifungal and antibacterial activities which are higher than those of the Schiff bases.

Some di- and triorganotin(IV) complexes of thio Schiff bases derived from the condensation of 2-amino-4-phenylthiazole, thiosemicarbazide and 2-amino-5-(o-anisyl)-1,3,4-thiadiazole with different aldehydes and ketones have also been tested for herbicidal, insecticidal and fungicidal activities, and against animal freeliving nematode. The results (36) indicated that only a few complexes were found to be active against various important herbicide and fungicide species. None of the complexes were found to be active against insects and freeliving nematode.

\section{REFERENCES}

1. R. H. Holm, Inorganic Biochemistry, Ed. G. L. Eichorn, Elsevier, Amsterdam, 2, 1137 (1974).

2. K. C. Joshi, V. N. Pathak and P. Panwar, Agric. Biol. Chem., 41, 543 (1977).

3 L. Albanus, N. E. Bjorklund, B. Gustafsson and M. Johsson, Acta Pharmacol. Toxicol. Suppl, 36, 93 (1975). 
4 I. Belsky, D. Gerther and A. Zilkha, J. Med. Chem., 11, 92 (1968).

5. R. C. Mehrotra, G. Srivastava and B. S. Saraswat, Revs. Si, Ge, Sn, Pb Compounds, 6, 171 (1982) (References therein).

6. G. E. Matsubayashi, M. Okunaka and T. Tanaka, J. Organomet. Chem., 56, 215 (1973).

7. G. E. Matsubayashi, T. Tanaka, S. Nishigaki and K. Nakatsu, J. Chem. Soc. Dalton, 501 (1979).

8. B. S. Saraswat, G. Srivastava and R. C. Mehrotra, J. Organomet. Chem., 164, 153 (1979).

9. B. S. Saraswat, Ph.D. Thesis, University of Rajasthan, Jaipur, (1977).

10. B. S. Saraswat, G. Srivastava and R. C. Mehrotra, Inorg. Nucl. Chem. Lett., 12, 235 (1976).

11. T. N. Srivastava and A. K. S. Chauhan, Abstr. 63' Session Ind. Sci. Congress, 16 (P) (1976).

12. T. N. Srivastava and A. K. S. Chauhan, J. Inorg. Nucl. Chem., 39, 371 (1977).

13. B. S. Saraswat, G. Srivastava and R. C. Mehrotra, Inorg. Chim. Acta, 36, 289 (1979).

14. T. N. Srivastava and A. K. S. Chauhan, Synth. React. Inorg. Met.-Org. Chem., 7, 373 (1977).

15. M. Nath, N. Sharma and C. L. Shanna, Synth. React. Inorg. Met.-Org. Chem., 21, 51 (1991).

16. T. N. Srivastava, A. K. S. Chauhan and V. K. Awasthi, Ind. J. Chem., 15A, 1023 (1977).

17. T. N. Srivastava, A. K. S. Chauhan and M. Agarwal, J. Inorg. Nucl. Chem., 41, 896 (1979).

18. B. S. Saraswat, G. Srivastava and R. C. Mehrotra, J. Inorg. Nucl. Chem., 43, 3003 (1981).

19. G. Faraglia, F. Maggio, R. Cefalu, R. Bosco and R. Barbieri, Inorg. Nucl. Chem. Lett., 5, 177 (1969).

20. R. Barbieri, R. Cefalu, S. C. Chandra and R. H. Herber, J. Organomet. Chem., 32, 97 (1971).

21. A. Vanden Bergen, R. J. Cozens and K. S. Murray, J. Chem. Soc. A., 3060 (1970).

22. R. Barbieri, G. Alonzo, A. Silvestri, N. Burriesci, N. Bertazzi, G. C. Stocco and L. Pellerito, Gazz. Chim. Ital., 104, 885 (1974).

23. K. Kawakami, M. Miya-Uchi and T. Tanaka, J. Inorg. Nucl. Chem., 33, 3773 (1971).

24. P. R. Shukla, M. C. Sharma and G. Narain, Synth. React. Inorg. Met. -Org. Chem., 20, 583 (1990).

25. B. S. Saraswat, G. Srivastava, R. C. Mehrotra, G. Sawhney and J. S. Baijal, J. Inorg. Nucl. Chem., 42, 805 (1980).

26. L. E. Khoo, J. P. Charland, E. J. Gabe and F. E. Smith, Inorg. Chim. Acta, 128, 139 (1987).

27. L. E. Khoo and F. E. Smith, Polyhedron, 1, 213 (1982).

28. M. Nath, N. Sharma and C. L. Sharma, Synth. React. Inorg. Met. -Org. Chem., 19, 339 
(1989).

29. M. Nath, S. Goyal and C. L. Sharma, Synth. React. Inorg. Met. -Org. Chem., 25, 821 (1995).

30. G. Alonzo, R. Barbieri, N. Burriesci, R. Cefalu, A. Gianguzza, S. Hauser, L. Pellerito and A. Silvestri, Abstr. $6^{\text {th }}$ Int. Conf. Organomet. Chem., 189 (P) (1973).

31. L. Pellerito, R. Cefalu, A. Gianguzza and R. Barbieri, J. Organomet. Chem., 70, 303 (1974).

32. D. Cunningham and M. Little, J. Organomet. Chem., 142, C58 (1977).

33. D. Cunningham and M. Little, Chim. Acta, 32, L81 (1979).

34. B. Teo Soon, G. Teoh Siang and Y. Yeap Guan, Chem. Technol. Silicon Tin, Proc. Asian Network Anal. Inorg. Chem. Int. Chem. Conf. Silicon, Tin., I It 509 (P) (1992).

35. M. Nath and S. Goyal, Main Group Met. Chem., 16, 167 (1993).

36. S. Goyal, Ph.D. Thesis, University of Roorkee, Roorkee, (1995).

37. B. N. Ghose, Synth. React. Inorg. Met. -Org. Chem., 12, 835 (1982).

38. P. Dixit and J. P. Tandon, Ind. J. Chem., Sect. A, 27A, 153 (1988).

38. R. Verma, V. D. Gupta and R. C. Mehrotra Inorg. Nucl. Chem. Lett., 15, 371 (1979).

39. F. Maggio, R. Bosco, R. Cefalu and R. Barbieri, Inorg. Nucl. Chem. Lett., 4, 389 (1968).

40. F. Maggio, R. Cefalu, R. Bosco, F. Bonati and V. Romano, Atti. Acad. Sci. Lett. Arti Palermo, Part. I, 28, 5 (1969).

41. R. Cefalu, R. Bosco, F. Bonati, F. Maggio and R. Barbieri, Abstr. $4^{\text {th }}$ Int. Conf. Organomet. Chem., (T8) (1969).

42. R. Cefalu, R. Bosco, F. Bonati, F. Maggio and R. Barbieri, Z. Anorg. Allg. Chem., 376, 180 (1970).

43. R. Bosco, Abstr. Convengnu Su Composti di Coordinazione, Roma, CNR, (194) (1970).

45. K. Kawakami and T. Tanaka, J. Organomet. Chem., 44, 409 (1973).

46. K. Dey, J. Inorg. Nucl. Chem., 32, 3125 (1970).

47. J. N. R. Ruddick and J. R. Sams, J. Organomet. Chem., 60, 233 (1973).

48. K. Kawakami, M. Miya-Uchi and T. Tanaka, J. Organomet. Chem., 70, 67 (1974).

49. A. K. Varshney, S. Varshney and J. P. Tandon, J. Prakt. Chem., 331, 511 (1989).

50. P. Dixit and J. P. Tandon, J. Prakt. Chem., 331, 659 (1989).

51. M. Nath, N. Sharma and C. L. Sharma, Synth. React. Inorg. Met. -Org. Chem., 20, 623 (1990).

52. M. Nath, S. Goyal and C. L. Sharma, Main Group. Met. Chem., 18, 51 (1995)

53. A. Kumari, R. V. Singh and J. P. Tandon, Phosphorus Sulphur Silicon Relat. Elem., 66, 195 (1992).

54: T. N. Srivastava, A. K. S. Chauhan and M. Agarwal, Synth. React. Inorg. Met. -Org. Chem., 10, 29 (1980)

55. M. P. Degaonkar, S. Gopinathan and C. Gopinathan, Synth. React. Inorg. Met. -Org. Chem., 19, 613 (1989). 
56. M. Nath, N. Sharma and C. L. Sharma, Synth. React. Inorg. Met. -Org. Chem., 20, 1355 (1990).

57. S. Gopinathan, M. P. Degaonkar, A. M. Hundekar and C. Gopinathan, Synth. React. Inorg. Met. -Org. Chem., 22, 1591 (1992).

58. S. Gopinathan, M. P. Degaonkar and C. Gopinathan, Appl. Organomet. Chem., 6, 69 (1992).

59. K. Dey, S. Ray and D. Bandyopadhyay, Proc. Natl. Acad. Sci., India Sect.A., 59, 367 (1989).

60. B. S. Saraswat. G. Srivastava and R. C. Mehrotra, Organomet. Chem., 129, 155 (1977).

61. K. Dey, S. Ray and D. Koner, Proc. Ind. Acad. Sci. (Chem. Sci.), 92, 257 (1983).

62. K. Dey, S. Ray and D. Bandyopadhyay, Proc. Natl. Acad. Sci., India, Sect. A.. 59, 179 (1989).

63. H. P. S. Chauhan, A. Bhargava and R. J. Rao, Ind. J. Chem., 32A, 157 (1993).

64. J. Wang, Y. Zhang, Y. Xu, Z. Wang and H. Youji, Heteroat. Chem., 13, 289 (1993).

65. J. Wang, Y. Zhang, Y. Xu and Z. Wang, Heteroat. Chem., 3, 599 (1992).

66. G. Alonzo, F. Di Bianca, A. Silvestri, G. C. Stocco, L. Pellerito, R. Cefalu, N. Bertazzi and R. Barbieri, Proc. 16 $6^{\text {th }}$ Int. Conf. Coord. Chem., 2, 20a (P) (1974).

67. L. Pellerito, R. Cefalu, A. Silvestri and R. Barbieri, Atti. Acad. Sci. Lett. Arti Palermo. Part 1., 33, 23 (1974).

68. L. Pellerito, R. Cefalu, A. Silvestri, F. Di Bianca, R. Barbieri, H. J. Haupt, H. Preut and F. Huber, J. Organomet. Chem., 78, 101 (1974).

69. P. F. R. Ewings, P. G. Harrison and A. Mangia, J. Organomet. Chem., 114, 35 (1976).

70. B. S. Saraswat, G. Srivastava and R. C. Mehrotra, J. Organomet. Chem., 137, 301 (1977).

71. R. Tiwari, G. Srivastava, R. C. Mehrotra and A. J. Crowe, Inorg. Chim. Acta, 111, 167 (1986).

72. A. K. Saxena, J. K. Koacher and J. P. Tandon. Inorg. Nucl. Chem. Lett., 17, 229 (1981).

73. A. Saxena and J. P. Tandon, Polyhedron., 3, 681 (1984).

74. A. Saxena and J. P. Tandon, Polyhedron, 2, 443 (1983).

75. S. K. Pandit, S. Gopinathan and C. Gopinathan, Ind. J. Chem., 21A, 1004 (1982).

76. S. A. Pardhy, S. Gopinathan and C. Gopinathan, Ind. J. Chem., 19A, 130 (1980).

77. G. K. Sandhu and S. S. Sandhu (Jr), Synth. React. Inorg. Met. -Org. Chem., 12, 215 (1982).

78. A. Varshney and J. P. Tandon, Proc. Ind. Acad. Sci., (Chem. Sci.), 94, 509 (1985).

79. A. Saxena, J. P. Tandon and A. J. Crowe, Polyhedron, 4. 1085 (1985)

80. M. Nath. C. L. Sharma and N. Sharma, Synth. React. Inorg. Met.-Org. Chem., 21, 807 (1991).

81. R. Cefalu, L. Pellerito and R. Barbieri, J Organumet. Chem., 32, 107 (1971).

82. R. Barbieri, R. H. Herber and S. C. Chandra, Abstr. $5^{\text {th }}$ Int. Conf. Organomet. Chem. 
278 (P) (1971).

83. R. Barbieri and R. H. Herber, J. Organomet. Chem., 42, 65 (1972).

84. P. J. Smith and D. Dodd, J. Organomet. Chem., 32, 195 (1971).

85. R. Barbieri, L. Pellerito, N. Bertazzi and G. C. Stocco, Inorg. Chim. Acta, 11, 173 (1974).

86. J. N. R. Ruddick, Revs. Si, Ge, Sn, Pb, Compounds, 2, 115 (1976).

87. L. Randaccio, J. Organomet. Chem., 55, C58 (1973).

88. R. Barbieri and G. C. Stocco, Gazz. Chim. Ital., 104, 149 (1974).

89. M. Calligaris, L. Randaccio, R. Barbieri and L. Pellerito, J. Organomet. Chem., 76, C56 (1974).

90. M. E. Kamwaya and L. E. Khoo, Acta crystallogr. Sect. C: Cryst. Struct. Commun., 41, 1027 (1985).

91. R. H. Herber and R. Barbieri, Gazz. Chim. Ital., 101, 149 (1971).

92. H. Preut, F. Huber, H. J. Haupt, R. Cefalu and R. Barbieri, Z. Anorg. Altg. Chem., 410, 88 (1974).

93. D. L. Evans and B. R. Penfold, J. Cryst. Mol. Struct., 5, 93 (1975).

94. H. Preut, F. Huber, R. Barbieri and N. Bertazzi, Z. Anorg. Allg. Chem., 423, 75 (1976).

95. H. Preut. H. J. Haupt, F. Huber, R. Cefalu and R. Barbieri, Z. Anorg. Allg. Chem., 407, 257 (1974).

96. M. Calligaris, G. Nardin and L. Randaccio, J. Chem. Soc. Dalton, 2003 (1972).

97. J. N. R. Ruddick and J. R. Sams, J. Chem. Soc. Dalton, 470 (1974).

98. J. N. R. Ruddick and J. R. Sams, Abstr. 6 $6^{\text {th }}$ Int. Conf. Organomet. Chem., 178 (P) (1973).

99. M. Nath, N. Sharma and C. L. Sharma, Thermochim. Acta, 149, 173 (1989).

100. M. Nath and N. Sharma, J. Ind. Chem. Soc., 72, 115 (1995).

101. N. Sharma, Ph.D. Thesis, University of Roorkee, Roorkee (1990) (References therein).

102. A. K. Saxena, J. K. Koacher, J. P. Tandon and S. R. Das, J. Toxicol. Environ. Health, 10, 709 (1982)

Received: September 12, 1995 - Accepted: October 13, 1995 Received in revised camera-ready format: December 4, 1995 